\title{
A Navier boundary value problem for Willmore surfaces of revolution*
}

\author{
Klaus Deckelnick ${ }^{\dagger}$ and Hans-Christoph Grunau ${ }^{\ddagger}$ \\ Fakultät für Mathematik \\ Otto-von-Guericke-Universität \\ Postfach 4120 \\ D-39016 Magdeburg
}

Dedicated to Prof. E. Heinz on the occasion of his 85th birthday

\begin{abstract}
We study a boundary value problem for Willmore surfaces of revolution, where the position and the mean curvature $H=0$ are prescribed as boundary data. The latter is a natural datum when considering critical points of the Willmore functional in classes of functions where only the position at the boundary is fixed.

For specific boundary positions, catenoids and a suitable part of the Clifford torus are explicit solutions. Numerical experiments, however, suggest a much richer bifurcation diagram. In the present paper we verify analytically some properties of the expected bifurcation diagram. Furthermore, we present a finite element method which allows the calculation of critical points of the Willmore functional irrespective of their stability properties.
\end{abstract}

\section{Introduction}

Recently, the Willmore functional and the associate $L^{2}$-gradient flow, the so-called Willmore flow, have attracted a lot of attention. Given a smooth immersed surface $f: M \rightarrow \mathbb{R}^{3}$, the Willmore functional is defined by

$$
W(f):=\int_{f(M)} H^{2} d S
$$

where $H=\left(\kappa_{1}+\kappa_{2}\right) / 2$ denotes the mean curvature of $\mathcal{R}:=f(M)$. Apart from being of geometric interest, the functional $W$ is a model for the elastic energy of thin shells or biological membranes. In these applications one is usually concerned with minima, or more generally with critical points of the Willmore functional. It is well-known that the corresponding surface $\mathcal{R}$ has to satisfy the Willmore equation

$$
\Delta_{\mathcal{R}} H+2 H\left(H^{2}-K\right)=0 \quad \text { on } \mathcal{R},
$$

where $\Delta_{\mathcal{R}}$ denotes the Laplace-Beltrami operator on $\mathcal{R}$ and $K$ its Gauss curvature. A solution of (1) is called a Willmore surface.

Although introduced already in the 19th century (see e.g. [P]), it was Willmore's work [Wi] which popularised again the investigation of the Willmore functional. For information on historical details and modelling aspects we refer to Nitsche's survey article [Nit], for the derivation of (1)

\footnotetext{
*Financial support of Deutsche Forschungsgemeinschaft (DFG) is gratefully acknowledged

${ }^{\dagger}$ e-mail: Klaus.Deckelnick@ovgu.de

${ }^{\ddagger}$ e-mail: Hans-Christoph.Grunau@ovgu.de
} 
as Euler-Lagrange equation of the Willmore functional cf. also [Th, p. 56]. Existence of closed Willmore surfaces of prescribed genus was proved by Simon [Sn] and Bauer \& Kuwert [BK]. Constrained closed Willmore surfaces of fixed conformal class were studied e.g. by Kuwert-Schätzle and Leschke-Pedit-Pinkall [KS1, KS2, LPP]. Rivière [R] obtained a far reaching regularity result.

If one is interested in surfaces with boundaries, then appropriate boundary conditions have to be added to (1). Since this equation is of fourth order one requires two sets of conditions and a discussion of possible choices can be found in [Nit] along with corresponding existence results. These results, however, are based on perturbation arguments and hence require severe smallness conditions on the data, which are by no means explicit. Existence results which do not require a smallness assumption on the data have been obtained for the Dirichlet problem where the position and the tangent space of the unknown Willmore surface are prescribed on the boundary. We refer to [DDG, DFGS] for the existence of Willmore surfaces of revolution in the case of symmetric data. Schätzle $[\mathrm{Sch}]$ proved an important general result concerning existence of branched Willmore immersions in $\mathbb{S}^{n}$ with boundary.

In the present paper we initiate the study of a different boundary value problem, the so-called Navier boundary value problem, in which the position and the mean curvature on the boundary are prescribed. In order to simplify the situation, we shall restrict ourselves to surfaces of revolution which are obtained by rotating the graph of a smooth positive function $u:[-1,1] \rightarrow(0, \infty)$ around the $x$-axis. For such a surface $\mathcal{R}$ the Willmore functional then reads (cf. Appendix A)

$$
W(u)=\int_{\mathcal{R}} H^{2} d S=\frac{\pi}{2} \int_{-1}^{1}\left(\frac{1}{u(x) \sqrt{1+u^{\prime}(x)^{2}}}-\frac{u^{\prime \prime}(x)}{\left(1+u^{\prime}(x)^{2}\right)^{3 / 2}}\right)^{2} u(x) \sqrt{1+u^{\prime}(x)^{2}} d x .
$$

In Lemma 6 we shall show that the first variation of $W$ in the direction of a function $\varphi \in H^{2} \cap$ $H_{0}^{1}(-1,1)$ is given by

$$
\left.\frac{d}{d t} W(u+t \varphi)\right|_{t=0}=-2 \pi\left[H \frac{u \varphi^{\prime}}{1+\left(u^{\prime}\right)^{2}}\right]_{-1}^{1}-2 \pi \int_{-1}^{1} u \varphi\left(\Delta_{\mathcal{R}} H+2 H\left(H^{2}-K\right)\right) d x .
$$

Hence, if $\alpha>0$ is given and $u \in C^{4}([-1,1],(0, \infty))$ is a critical point of the Willmore functional $W$ in $\{\alpha\}+\left(H^{2}(-1,1) \cap H_{0}^{1}(-1,1)\right)$, then $u$ is a solution of the following boundary value problem:

$$
\left\{\begin{array}{l}
\Delta_{\mathcal{R}} H+2 H\left(H^{2}-K\right)=0 \quad \text { in }(-1,1) \\
u( \pm 1)=\alpha, \quad H( \pm 1)=0
\end{array}\right.
$$

Observing (5) and (6) below we see that the differential equation in (4) is a highly nonlinear ODE of fourth order; note that the boundary condition $H( \pm 1)=0$ is nonlinear as well.

If the equation $\alpha=\frac{\cosh (b)}{b}$ has a positive solution, then the catenoid generated by the function

$$
u_{b}(x):=\frac{1}{b} \cosh (b x)
$$

satisfies $H \equiv 0$ and therefore yields a solution of (4). Furthermore, a discussion of the function $b \mapsto \cosh (b) / b$ shows that there exists $\alpha_{0}>0$ such that (4) has two catenoid solutions for $\alpha>\alpha_{0}$, one such solution for $\alpha=\alpha_{0}$, while for $\alpha \in\left(0, \alpha_{0}\right)$ the catenoid solutions cease to exist, see Figure 1. Here, $\alpha_{0}:=\cosh \left(b_{0}\right) / b_{0}=1.5088795 \ldots$, where $b_{0}=1.1996786 \ldots$ is the solution of the equation $\cosh \left(b_{0}\right)=b_{0} \sinh \left(b_{0}\right)$. Moreover, it is shown in [DHKW, Chapter 6.1, Theorem 3] that (4) does not have a connected minimal surface solution at all - whether symmetric or not - for $\alpha<1$. However, for $\alpha=1$ there is another explicitly known solution of (4), namely the part of the Clifford torus that is obtained by rotating the graph of the function $[-1,1] \ni x \mapsto u(x)=2-\sqrt{2-x^{2}}$, see Figure 2. 

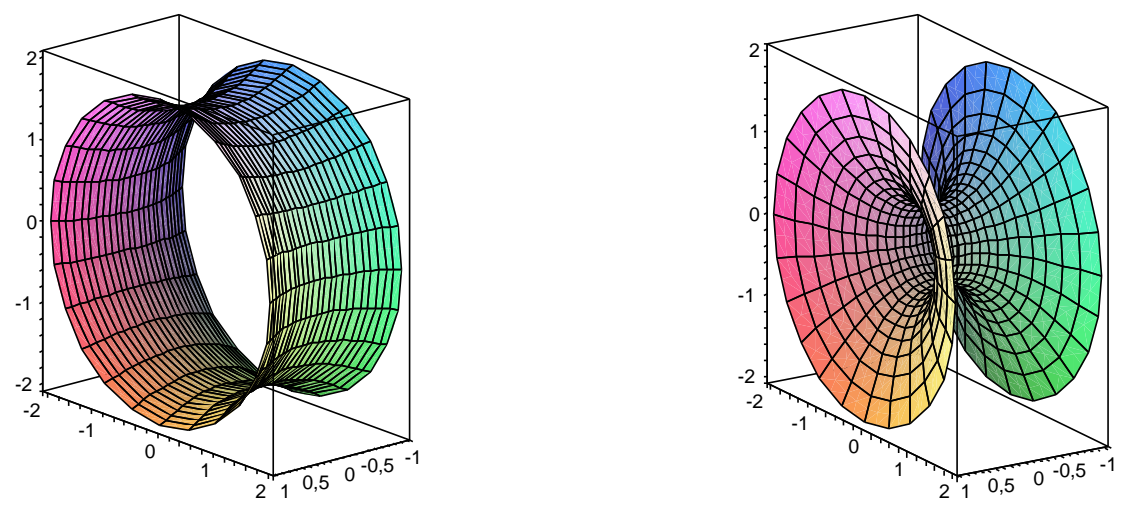

Figure 1: Catenoid solutions of the boundary value problem (4) for $\alpha=2$.

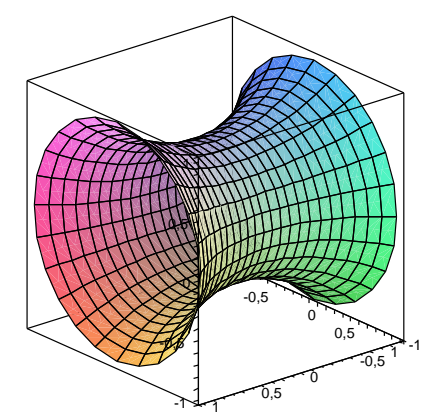

Figure 2: Part of the Clifford torus solving (4) with $\alpha=1$.

Numerical experiments carried out by Fröhlich [F] and Kastsian [Ka] suggest that this solution is not isolated but rather belongs to a branch of solutions. This branch seems to comprise solutions of (4) for positive values of $\alpha$ beyond $\alpha_{0}$ that are not minimal surfaces unless $\alpha=\alpha_{0}$. Hence it is natural to expect that this branch bifurcates in $\alpha_{0}$ from the branch of minimal surfaces described above, see Figure 3. In the current work we attempt to rigorously verify at least part of this diagram. Our first main result shows that the Clifford torus is not an isolated solution of (4) but belongs to a branch of solutions.

Theorem 1. There exists $\varepsilon_{0}>0$ such that for $\alpha \in\left(1-\varepsilon_{0}, 1+\varepsilon_{0}\right)$, the boundary value problem (4) has a solution $u \in C^{\infty}([-1,1],(0, \infty))$.

Remark 1. Numerical evidence suggests that this solution branch can be extended towards $\alpha \searrow 0$ and that the solutions converge to the unit sphere as $\alpha \searrow 0$. For Dirichlet boundary conditions the analogous result is rigorously proved in [DFGS].

Our second main result concerns the existence of the expected bifurcation point on the branch of catenoid solutions. For technical reasons it is more suitable to choose the central value $u(0)$ as bifurcation parameter $\lambda$.

Theorem 2. For $\lambda>0$ let $u_{\lambda}(x)=\lambda \cosh \left(\frac{x}{\lambda}\right)$ denote the minimal surface solutions of (4) with $\alpha=\alpha(\lambda)=\lambda \cosh \left(\frac{1}{\lambda}\right)$. Let $b_{0}=1.1996786 \ldots$ be the unique positive solution of the equation 
$\cosh (b)=b \sinh (b), \lambda_{0}=1 / b_{0}=0.83355 \ldots$ The parameter $\lambda_{0}$ corresponds to $\alpha_{0}=\cosh \left(b_{0}\right) / b_{0}=$ $1.5088795 \ldots$ Then $\left(\alpha_{0}, u_{\lambda_{0}}\right)$ is the unique bifurcation point for (4) on the branch $\lambda \mapsto\left(\alpha(\lambda), u_{\lambda}\right)$.

Our paper is organised as follows: In Section 2 we give the proof of Theorem 1 via an implicit function theorem, while Theorem 2 is proved in Section 3. Section 4 describes a numerical method in order to find approximate solutions of (4). In Appendices A and B we present detailed calculations concerning the first and second variation of the Willmore functional and the solution space of the linearisation of the Willmore equation around the Clifford torus, respectively.

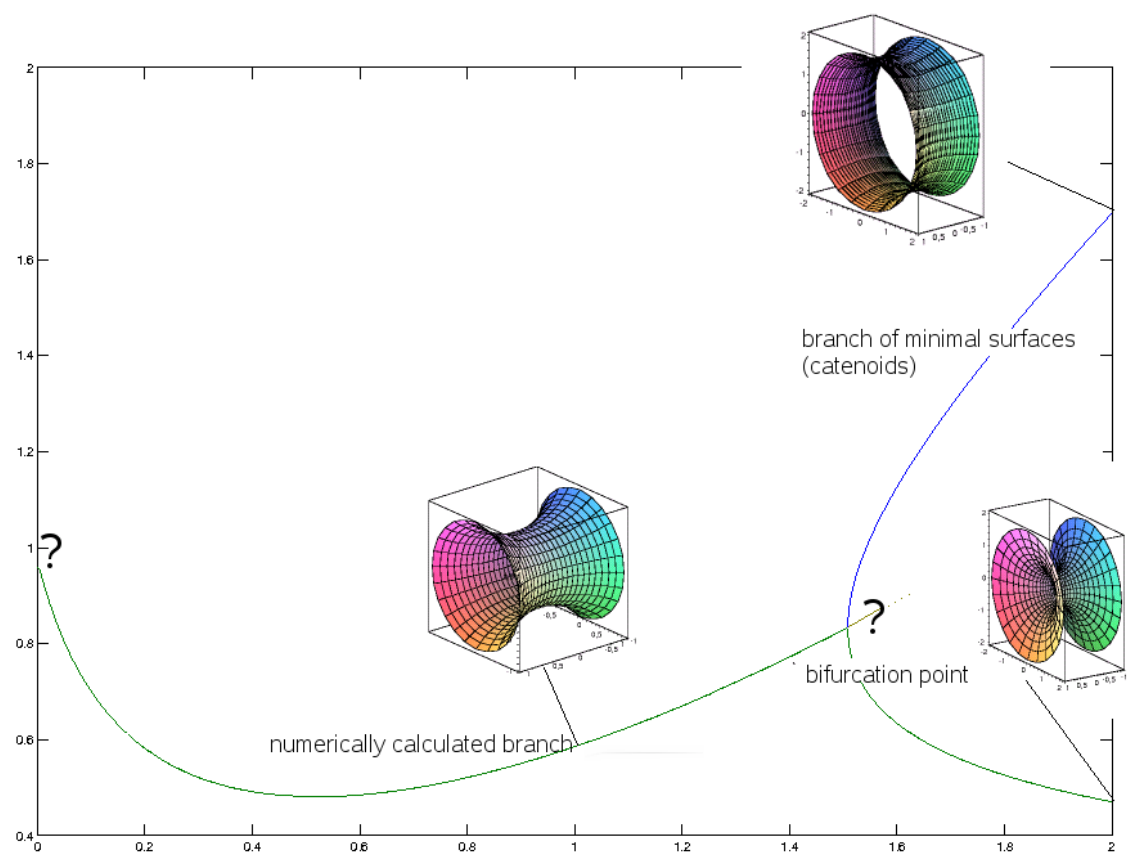

Figure 3: Numerically calculated branches of solutions to (4): We display the central value $u(0)$ over the boundary datum $\alpha=u( \pm 1)$.

\section{Linearisation around the Clifford torus}

Let us consider a surface of revolution generated by the graph of a sufficiently smooth function $u:[-1,1] \rightarrow(0, \infty)$ as follows:

$$
\mathcal{R}:(x, \varphi) \mapsto(x, u(x) \cos \varphi, u(x) \sin \varphi), \quad x \in[-1,1], \varphi \in[0,2 \pi] .
$$

It is not difficult to verify that the first and second fundamental forms are given by

$$
\left(g_{i j}\right)=\left(\begin{array}{cc}
1+u^{\prime}(x)^{2} & 0 \\
0 & u(x)^{2}
\end{array}\right), \quad\left(L_{i j}\right)=\frac{1}{\sqrt{1+u^{\prime}(x)^{2}}}\left(\begin{array}{cc}
-u^{\prime \prime}(x) & 0 \\
0 & u(x)
\end{array}\right),
$$

while we have for the mean curvature $H$ and the Gauss curvature $K$ the formulae

$$
H=-\frac{u^{\prime \prime}(x)}{2\left(1+u^{\prime}(x)^{2}\right)^{3 / 2}}+\frac{1}{2 u(x) \sqrt{1+u^{\prime}(x)^{2}}}, \quad K=-\frac{u^{\prime \prime}(x)}{u(x)\left(1+u^{\prime}(x)^{2}\right)^{2}} .
$$


Here we use the sign convention that $H$ is positive if the surface is mean convex with respect to the inner normal. Furthermore,

$$
\Delta_{\mathcal{R}} H=\frac{1}{u(x) \sqrt{1+u^{\prime}(x)^{2}}} \cdot \partial_{x}\left(\frac{u(x)}{\sqrt{1+u^{\prime}(x)^{2}}} \partial_{x}\left(\frac{1}{2 u(x) \sqrt{1+u^{\prime}(x)^{2}}}-\frac{u^{\prime \prime}(x)}{2\left(1+u^{\prime}(x)^{2}\right)^{3 / 2}}\right)\right) .
$$

As a consequence, the value of the Willmore functional for a surface of revolution is given by

$$
\tilde{W}(u):=\frac{1}{\pi} W(u)=\frac{1}{2} \int_{-1}^{1}\left(\frac{1}{u(x) \sqrt{1+u^{\prime}(x)^{2}}}-\frac{u^{\prime \prime}(x)}{\left(1+u^{\prime}(x)^{2}\right)^{3 / 2}}\right)^{2} u(x) \sqrt{1+u^{\prime}(x)^{2}} d x .
$$

In this section we always consider the part of the Clifford torus $u(x)=2-\sqrt{2-x^{2}}$, solving (4) with $\alpha=1$. Clearly,

$$
u^{\prime}(x)=x\left(2-x^{2}\right)^{-1 / 2}, \quad u^{\prime \prime}(x)\left(1+u^{\prime}(x)^{2}\right)^{-3 / 2}=1 / \sqrt{2},
$$

so that it is straightforward to verify that $u$ is a solution of (4). In order to show that (4) has also solutions for $\alpha$ close to 1 we use the implicit function theorem which requires the analysis of the the linearisation of (4) in $u$.

Lemma 1. The second variation of $\tilde{W}$ in $u(x)=2-\sqrt{2-x^{2}}$ is given by

$$
\begin{aligned}
\tilde{W}^{\prime \prime}(u)(\varphi, \eta)= & \int_{-1}^{1} \frac{u(x) \varphi^{\prime \prime}(x) \eta^{\prime \prime}(x)}{\left(1+u^{\prime}(x)^{2}\right)^{5 / 2}} d x \\
& +\int_{-1}^{1} \frac{\varphi^{\prime}(x) \eta^{\prime}(x)}{u(x)\left(1+u^{\prime}(x)^{2}\right)^{5 / 2}}\left(6-\frac{20}{\left(2-x^{2}\right)^{1 / 2}}+\frac{6}{2-x^{2}}+\frac{12}{\left(2-x^{2}\right)^{3 / 2}}\right) d x \\
& +\int_{-1}^{1} \frac{\sqrt{2} \varphi(x) \eta(x)}{u(x)^{2}} d x+\left[\frac{3 u^{\prime}(x) \varphi^{\prime}(x) \eta^{\prime}(x)}{\left(1+u^{\prime}(x)^{2}\right)^{5 / 2}}-\frac{5 u^{\prime}(x) \varphi^{\prime}(x) \eta^{\prime}(x)}{\sqrt{2}\left(1+u^{\prime}(x)^{2}\right)^{2}}\right]_{-1}^{1},
\end{aligned}
$$

where $\varphi, \eta \in H^{2} \cap H_{0}^{1}(-1,1)$.

Proof. We make use of the expression for the second variation derived in Lemma 6 of Appendix A and begin by rewriting the integrals involving $(\varphi \eta)^{\prime},(\varphi \eta)^{\prime \prime}$ and $\left(\varphi^{\prime} \eta^{\prime}\right)^{\prime}$. To do so we use $(7)$ together with the fact that $u( \pm 1)=1$. Integration by parts yields:

$$
\begin{aligned}
\int_{-1}^{1} \frac{u^{\prime}(\varphi \eta)^{\prime}}{u^{2}\left(1+\left(u^{\prime}\right)^{2}\right)^{3 / 2}} d x= & -\int_{-1}^{1} \frac{u^{\prime \prime} \varphi \eta}{u^{2}\left(1+\left(u^{\prime}\right)^{2}\right)^{3 / 2}} d x \\
& +2 \int_{-1}^{1} \frac{\left(u^{\prime}\right)^{2} \varphi \eta}{u^{3}\left(1+\left(u^{\prime}\right)^{2}\right)^{3 / 2}} d x+3 \int_{-1}^{1} \frac{\left(u^{\prime}\right)^{2} u^{\prime \prime} \varphi \eta}{u^{2}\left(1+\left(u^{\prime}\right)^{2}\right)^{5 / 2}} d x \\
= & -\frac{1}{\sqrt{2}} \int_{-1}^{1} \frac{\varphi \eta}{u^{2}} d x+2 \int_{-1}^{1} \frac{\left(u^{\prime}\right)^{2} \varphi \eta}{u^{3}\left(1+\left(u^{\prime}\right)^{2}\right)^{3 / 2}} d x \\
& +\frac{3}{\sqrt{2}} \int_{-1}^{1} \frac{\left(u^{\prime}\right)^{2} \varphi \eta}{u^{2}\left(1+\left(u^{\prime}\right)^{2}\right)} d x \\
\int_{-1}^{1} \frac{u^{\prime \prime}(\varphi \eta)^{\prime \prime}}{\left(1+\left(u^{\prime}\right)^{2}\right)^{5 / 2}} d x= & \frac{1}{\sqrt{2}} \int_{-1}^{1} \frac{(\varphi \eta)^{\prime \prime}}{1+\left(u^{\prime}\right)^{2}} d x=\sqrt{2} \int_{-1}^{1} \frac{u^{\prime} u^{\prime \prime}(\varphi \eta)^{\prime}}{\left(1+\left(u^{\prime}\right)^{2}\right)^{2}} d x \\
= & \int_{-1}^{1} \frac{u^{\prime}(\varphi \eta)^{\prime}}{\left(1+\left(u^{\prime}\right)^{2}\right)^{1 / 2}} d x=-\int_{-1}^{1} \frac{u^{\prime \prime} \varphi \eta}{\left(1+\left(u^{\prime}\right)^{2}\right)^{3 / 2}} d x=-\frac{1}{\sqrt{2}} \int_{-1}^{1} \varphi \eta d x \\
\int_{-1}^{1} \frac{u^{\prime}\left(u^{\prime \prime}\right)^{2}(\varphi \eta)^{\prime}}{\left(1+\left(u^{\prime}\right)^{2}\right)^{7 / 2}} d x= & \frac{1}{2} \int_{-1}^{1} \frac{u^{\prime}(\varphi \eta)^{\prime}}{\left(1+\left(u^{\prime}\right)^{2}\right)^{1 / 2}} d x=-\frac{1}{2 \sqrt{2}} \int_{-1}^{1} \varphi \eta d x
\end{aligned}
$$




$$
\begin{aligned}
\int_{-1}^{1} \frac{u u^{\prime} u^{\prime \prime}\left(\varphi^{\prime} \eta^{\prime}\right)^{\prime}}{\left(1+\left(u^{\prime}\right)^{2}\right)^{7 / 2}} d x= & \frac{1}{\sqrt{2}} \int_{-1}^{1} \frac{u u^{\prime}\left(\varphi^{\prime} \eta^{\prime}\right)^{\prime}}{\left(1+\left(u^{\prime}\right)^{2}\right)^{2}} d x \\
= & -\frac{1}{\sqrt{2}} \int_{-1}^{1} \frac{\left(u^{\prime}\right)^{2} \varphi^{\prime} \eta^{\prime}}{\left(1+\left(u^{\prime}\right)^{2}\right)^{2}} d x-\frac{1}{\sqrt{2}} \int_{-1}^{1} \frac{u u^{\prime \prime} \varphi^{\prime} \eta^{\prime}}{\left(1+\left(u^{\prime}\right)^{2}\right)^{2}} d x \\
& +2 \sqrt{2} \int_{-1}^{1} \frac{u\left(u^{\prime}\right)^{2} u^{\prime \prime} \varphi^{\prime} \eta^{\prime}}{\left(1+\left(u^{\prime}\right)^{2}\right)^{3}} d x+\frac{1}{\sqrt{2}}\left[\frac{u^{\prime} \varphi^{\prime} \eta^{\prime}}{\left(1+\left(u^{\prime}\right)^{2}\right)^{2}}\right]_{-1}^{1} \\
= & -\frac{1}{\sqrt{2}} \int_{-1}^{1} \frac{\left(u^{\prime}\right)^{2} \varphi^{\prime} \eta^{\prime}}{\left(1+\left(u^{\prime}\right)^{2}\right)^{2}} d x-\frac{1}{2} \int_{-1}^{1} \frac{u \varphi^{\prime} \eta^{\prime}}{\left(1+\left(u^{\prime}\right)^{2}\right)^{1 / 2}} d x \\
& +2 \int_{-1}^{1} \frac{u\left(u^{\prime}\right)^{2} \varphi^{\prime} \eta^{\prime}}{\left(1+\left(u^{\prime}\right)^{2}\right)^{3 / 2}} d x+\frac{1}{\sqrt{2}}\left[\frac{u^{\prime} \varphi^{\prime} \eta^{\prime}}{\left(1+\left(u^{\prime}\right)^{2}\right)^{2}}\right]_{-1}^{1} .
\end{aligned}
$$

Collecting terms and exploiting again (7) we conclude:

$$
\begin{aligned}
\tilde{W}^{\prime \prime}(u)(\varphi, \eta)= & \int_{-1}^{1} \varphi \eta\left(\frac{1}{u^{3}\left(1+\left(u^{\prime}\right)^{2}\right)^{1 / 2}}-\frac{1}{2 \sqrt{2} u^{2}}+\frac{\left(u^{\prime}\right)^{2}}{u^{3}\left(1+\left(u^{\prime}\right)^{2}\right)^{3 / 2}}\right) d x \\
& +\int_{-1}^{1} \varphi \eta\left(\frac{3\left(u^{\prime}\right)^{2}}{2 \sqrt{2} u^{2}\left(1+\left(u^{\prime}\right)^{2}\right)}+\frac{\sqrt{2}}{8}\right) d x \\
& +\int_{-1}^{1} \varphi^{\prime} \eta^{\prime}\left(\frac{-1}{2 u\left(1+\left(u^{\prime}\right)^{2}\right)^{3 / 2}}+\frac{3\left(u^{\prime}\right)^{2}}{2 u\left(1+\left(u^{\prime}\right)^{2}\right)^{5 / 2}}\right) d x \\
& +\int_{-1}^{1} \varphi^{\prime} \eta^{\prime}\left(-\frac{\sqrt{2}}{1+\left(u^{\prime}\right)^{2}}+\frac{5\left(u^{\prime}\right)^{2}}{\sqrt{2}\left(1+\left(u^{\prime}\right)^{2}\right)^{2}}+\frac{5 u}{4\left(1+\left(u^{\prime}\right)^{2}\right)^{3 / 2}}\right) d x \\
& +\int_{-1}^{1} \varphi^{\prime \prime} \eta^{\prime \prime} \frac{u}{\left(1+\left(u^{\prime}\right)^{2}\right)^{5 / 2}} d x+\left[\frac{3 u^{\prime} \varphi^{\prime} \eta^{\prime}}{\left(1+\left(u^{\prime}\right)^{2}\right)^{5 / 2}}-\frac{5 u^{\prime} \varphi^{\prime} \eta^{\prime}}{\sqrt{2}\left(1+\left(u^{\prime}\right)^{2}\right)^{2}}\right]_{-1}^{1}
\end{aligned}
$$

Making again use of $u(x)=2-\sqrt{2-x^{2}}$ and (7), the claim follows by elementary calculations.

In explicit terms, the linearisation of the boundary value problem (4) for the Willmore equation around the Clifford torus is given by

$$
\left\{\begin{array}{l}
\frac{d^{2}}{d x^{2}}\left(c(x) \varphi^{\prime \prime}(x)\right)-\frac{d}{d x}\left(b(x) \varphi^{\prime}(x)\right)+a(x) \varphi(x)=0 \quad \text { for } x \in(-1,1) \\
\varphi(1)=\varphi(-1)=0, \\
\varphi^{\prime \prime}(1)-2 \varphi^{\prime}(1)=0, \quad \varphi^{\prime \prime}(-1)+2 \varphi^{\prime}(-1)=0
\end{array}\right.
$$

where the coefficients are defined as follows:

$$
\begin{aligned}
a(x) & :=\frac{8}{\left(2-\sqrt{2-x^{2}}\right)^{2}}, \\
b(x) & :=\frac{2\left(2-x^{2}\right)}{2-\sqrt{2-x^{2}}}\left(6+3\left(2-x^{2}\right)^{1 / 2}-10\left(2-x^{2}\right)+3\left(2-x^{2}\right)^{3 / 2}\right) \\
& =\frac{-8\left(2-x^{2}\right)}{2-\sqrt{2-x^{2}}}+10\left(2-x^{2}\right)+8\left(2-x^{2}\right)^{3 / 2}-6\left(2-x^{2}\right)^{2}, \\
c(x) & :=2\left(2-x^{2}\right)^{5 / 2}-\left(2-x^{2}\right)^{3}=\left(2-x^{2}\right)^{5 / 2}\left(2-\sqrt{2-x^{2}}\right) .
\end{aligned}
$$


Lemma 2. The general solution of the differential equation in (8) is given by the following fundamental system:

$$
\begin{aligned}
& \varphi_{1}(x)=\frac{x}{\sqrt{2-x^{2}}}, \\
& \varphi_{2}(x)=\frac{\sqrt{2-x^{2}}-1}{\sqrt{2-x^{2}}}, \\
& \varphi_{3}(x)=\frac{2-\sqrt{2-x^{2}}}{\sqrt{2-x^{2}}} \cos \left(\sqrt{2} \arctan \frac{x}{\sqrt{2}\left(\sqrt{2-x^{2}}-1\right)}\right), \\
& \varphi_{4}(x)=\frac{2-\sqrt{2-x^{2}}}{\sqrt{2-x^{2}}} \sin \left(\sqrt{2} \arctan \frac{x}{\sqrt{2}\left(\sqrt{2-x^{2}}-1\right)}\right) .
\end{aligned}
$$

Proof. See Appendix B.

Lemma 3. For the boundary value problem (8) we have uniqueness, i.e. the only solution of (8) is $\varphi(x) \equiv 0$.

Proof. Assume that $\varphi:(-1,1) \rightarrow \mathbb{R}$ is a solution of (8). According to Lemma 2, there exist constants $\beta_{1}, \ldots, \beta_{4} \in \mathbb{R}$ such that $\varphi(x)=\sum_{i=1}^{4} \beta_{i} \varphi_{i}(x), x \in[-1,1]$. Furthermore,

$$
\varphi(1)=0, \varphi(-1)=0 ; \varphi^{\prime \prime}(1)-2 \varphi^{\prime}(1)=0, \varphi^{\prime \prime}(-1)+2 \varphi^{\prime}(-1)=0 .
$$

The calculations following (32), (33) in Appendix B imply that $\beta_{i}=0$ for $i=1, \ldots, 4$ and hence $\varphi \equiv 0$.

As a consequence of the previous lemma we have that the linearisation of the Willmore equation around the Clifford torus is boundedly invertible in suitable function spaces. Employing the implicit function theorem, this gives rise to a local existence result for the Willmore boundary value problem (4) for data $\alpha$ close to 1 , which is the boundary datum of the Clifford torus. This means that we are now able to prove Theorem 1.

Theorem 1. There exists $\varepsilon_{0}>0$ such that for $\alpha \in\left(1-\varepsilon_{0}, 1+\varepsilon_{0}\right)$, the boundary value problem (4) has a solution $u \in C^{\infty}([-1,1],(0, \infty))$.

Proof. We apply the implicit function theorem as it can be found in [De, Theorem 15.1]. Let $X=\mathbb{R}, U=(0, \infty) \subset X, Y=C^{4}([-1,1]), V=C^{4}([-1,1],(0, \infty)) \subset Y, Z=C^{0}([-1,1]) \times \mathbb{R}^{4}$ as well as $F: V \rightarrow Z, G: U \times V \rightarrow Z$ with

$$
\begin{aligned}
F(u) & :=\left(\Delta_{\mathcal{R}} H+2 H\left(H^{2}-K\right), u(-1), u(1), H(-1), H(1)\right) ; \\
G(\alpha, u) & :=F(u)-\alpha(0,1,1,0,0) .
\end{aligned}
$$

We put $\alpha_{0}=1, u_{0}(x)=2-\sqrt{2-x^{2}}, x \in[-1,1]$ and

$$
L:=\frac{\partial G}{\partial u}\left(\alpha_{0}, u_{0}\right)=\frac{\partial F}{\partial u}\left(u_{0}\right)
$$

It is straightforward to see that $L$ allows for an elliptic theory. By means of the Fredholm alternative we see that bounded invertibility of $L$ follows from its injectivity. The latter is proved in Lemma 3. Hence, the implicit function theorem yields the claim, in a first step with $u \in$ $C^{4}([-1,1],(0, \infty))$. Since the Willmore equation is "autonomous" and only quasilinear we conclude that $u \in C^{\infty}([-1,1])$ by using standard bootstrapping arguments. 


\section{The bifurcation point}

The bifurcation point we want to verify in Problem (4) and Figure 3 resp. is also a turning point on the branch of minimal surfaces. In order not to verify just the latter fact we have to take a different point of view: Instead of (4), we consider

$$
\left\{\begin{array}{l}
\Delta_{\mathcal{R}} H+2 H\left(H^{2}-K\right)=0 \quad \text { in }(-1,1), \\
u(0)=\lambda, \quad u(x)=u(-x), \quad H( \pm 1)=0 .
\end{array}\right.
$$

We consider $\lambda$ as bifurcation parameter and the boundary values $\alpha=u( \pm 1)$ as a function of $\lambda$, i.e. we interchange the role of the axes in Figure 3. The "trivial" solution branch of (12) is given by the minimal surfaces $u_{\lambda}(x)=\frac{\cosh (b x)}{b}$ with $b=\frac{1}{\lambda}$. Since

$$
\begin{aligned}
\left.\frac{d}{d \varepsilon} H(u+\varepsilon \varphi)\right|_{\varepsilon=0}= & -\frac{\varphi^{\prime \prime}}{2\left(1+\left(u^{\prime}\right)^{2}\right)^{3 / 2}}-3 \frac{u^{\prime} \varphi^{\prime}}{1+\left(u^{\prime}\right)^{2}} H \\
& +\frac{u^{\prime} \varphi^{\prime}}{u\left(1+\left(u^{\prime}\right)^{2}\right)^{3 / 2}}-\frac{\varphi}{2 u^{2} \sqrt{1+\left(u^{\prime}\right)^{2}}},
\end{aligned}
$$

linearising the differential equation in (12) on minimal surfaces yields:

$$
\begin{aligned}
0= & \frac{1}{u \sqrt{1+\left(u^{\prime}\right)^{2}}} \\
& \cdot \partial_{x}\left(\frac{u}{\sqrt{1+\left(u^{\prime}\right)^{2}}} \partial_{x}\left(-\frac{\varphi^{\prime \prime}}{2\left(1+\left(u^{\prime}\right)^{2}\right)^{3 / 2}}+\frac{u^{\prime} \varphi^{\prime}}{u\left(1+\left(u^{\prime}\right)^{2}\right)^{3 / 2}}-\frac{\varphi}{2 u^{2} \sqrt{1+\left(u^{\prime}\right)^{2}}}\right)\right) \\
& -2 K\left(-\frac{\varphi^{\prime \prime}}{2\left(1+\left(u^{\prime}\right)^{2}\right)^{3 / 2}}+\frac{u^{\prime} \varphi^{\prime}}{u\left(1+\left(u^{\prime}\right)^{2}\right)^{3 / 2}}-\frac{\varphi}{2 u^{2} \sqrt{1+\left(u^{\prime}\right)^{2}}}\right) .
\end{aligned}
$$

Determining the null space of the linearisation of (12) around the solution $u=\frac{\cosh (b x)}{b}$ leads to the following boundary value problem for $\varphi$ :

$$
\left\{\begin{aligned}
0= & \partial_{x}^{2}\left(\frac{1}{2 \cosh (b x)^{4}}\left(\cosh (b x) \varphi^{\prime \prime}(x)-2 b \sinh (b x) \varphi^{\prime}(x)+b^{2} \cosh (b x) \varphi(x)\right)\right) \\
& +\frac{b^{2}}{\cosh (b x)^{6}}\left(\cosh (b x) \varphi^{\prime \prime}(x)-2 b \sinh (b x) \varphi^{\prime}(x)+b^{2} \cosh (b x) \varphi(x)\right), \quad x \in[-1,1], \\
\varphi(x)= & \varphi(-x), \quad x \in[-1,1], \\
0= & \varphi(0), \\
0= & \varphi^{\prime \prime}(1)-\frac{2 b \sinh (b)}{\cosh (b)} \varphi^{\prime}(1)+b^{2} \varphi(1) .
\end{aligned}\right.
$$

Lemma 4. The space of even solutions to the differential equation in (13) is spanned by

$$
\begin{aligned}
\varphi_{1}(x):= & \cosh (b x)-b x \sinh (b x) \\
\varphi_{2}(x):= & \sinh (b x)\left(\frac{5}{3} \cosh (b x) \sinh (b x)\right. \\
& \left.+\frac{5}{3} b x-2 b x \cosh (b x)^{2}+\frac{2}{3}(b x)^{2} \cosh (b x) \sinh (b x)-\frac{2}{9}(b x)^{3}\right) \\
& -(\cosh (b x)-b x \sinh (b x))\left(\cosh (b x)^{2}-\frac{2}{3} b x \sinh (b x) \cosh (b x)+\frac{1}{3}(b x)^{2}\right) .
\end{aligned}
$$

Proof. In order to determine the general even solution of the differential equation, we solve in a first step for $\Phi(x):=\cosh (b x) \varphi^{\prime \prime}(x)-2 b \sinh (b x) \varphi^{\prime}(x)+b^{2} \cosh (b x) \varphi(x)$. The general solution of $\partial_{x}^{2}\left(\frac{1}{2 \cosh (b x)^{4}} \Phi(x)\right)+\frac{b^{2}}{\cosh (b x)^{6}} \Phi(x)=0$, irrespective of whether it is even or not, is given by

$$
\Phi(x)=\beta_{1} \cosh (b x)^{3} \sinh (b x)+\beta_{2} \cosh (b x)^{3}(\cosh (b x)-b x \sinh (b x)), \quad \beta_{1}, \beta_{2} \in \mathbb{R} .
$$


Since the differential equation

$$
\cosh (b x) \varphi^{\prime \prime}(x)-2 b \sinh (b x) \varphi^{\prime}(x)+b^{2} \cosh (b x) \varphi(x)=\cosh (b x)^{3} \sinh (b x)
$$

has no even solution, we only need to consider:

$$
\cosh (b x) \varphi^{\prime \prime}(x)-2 b \sinh (b x) \varphi^{\prime}(x)+b^{2} \cosh (b x) \varphi(x)=\beta_{2} \cosh (b x)^{3}(\cosh (b x)-b x \sinh (b x)) .
$$

As for $\varphi_{2}$ we calculate:

$$
\begin{aligned}
\varphi_{2}(x)= & \frac{5}{3} \cosh (b x) \sinh (b x)^{2}+\frac{5}{3} b x \sinh (b x)-\frac{1}{3} b x \cosh (b x)^{2} \sinh (b x) \\
& +\frac{1}{9}(b x)^{3} \sinh (b x)-\cosh (b x)^{3}-\frac{1}{3}(b x)^{2} \cosh (b x), \\
\varphi_{2}^{\prime}(x)= & \frac{5}{3} b \cosh (b x)^{2} \sinh (b x)-b^{2} x \cosh (b x)^{3}+\frac{1}{9} b^{4} x^{3} \cosh (b x)+\frac{5}{3} b^{2} x \cosh (b x), \\
\varphi_{2}^{\prime \prime}(x)= & \frac{10}{3} b^{2} \cosh (b x) \sinh (b x)^{2}+\frac{2}{3} b^{2} \cosh (b x)^{3}-3 b^{3} x \cosh (b x)^{2} \sinh (b x) \\
& +\frac{1}{3} b^{4} x^{2} \cosh (b x)+\frac{1}{9} b^{5} x^{3} \sinh (b x)+\frac{5}{3} b^{2} \cosh (b x)+\frac{5}{3} b^{3} x \sinh (b x),
\end{aligned}
$$

hence

$$
\cosh (b x) \varphi_{2}^{\prime \prime}(x)-2 b \sinh (b x) \varphi_{2}^{\prime}(x)+b^{2} \cosh (b x) \varphi_{2}(x)=\frac{4}{3} b^{2} \cosh (b x)^{3}(\cosh (b x)-b x \sinh (b x)) .
$$

Because $\varphi_{1}$ and $x \mapsto \sinh (b x)$ solve the homogeneous equation, i.e. (14) with $\beta_{2}=0$, its general solution is given by

$$
x \mapsto \frac{3 \beta_{2}}{4 b^{2}} \varphi_{2}(x)+\beta_{3} \varphi_{1}(x)+\beta_{4} \sinh (b x),
$$

where $\beta_{3}, \beta_{4} \in \mathbb{R}$. Since we only need to consider even functions we may put $\beta_{4}=0$. So, the space of even solutions to the differential equation in (13) is spanned by $\varphi_{1}$ and $\varphi_{2}$.

Lemma 5. Let $b_{0}=1.1996786 \ldots$ be the unique positive solution of the equation $\cosh (b)=b \sinh (b)$. If $b \in(0, \infty) \backslash\left\{b_{0}\right\}$, the only solution of (13) is $\varphi(x) \equiv 0$. If $b=b_{0}$, then the null space of (13) is one-dimensional and spanned by

$$
\begin{aligned}
\varphi(x)= & \varphi_{1}(x)+\varphi_{2}(x) \\
= & \cosh \left(b_{0} x\right)-b_{0} x \sinh \left(b_{0} x\right)+\sinh \left(b_{0} x\right)\left(\frac{5}{3} \cosh \left(b_{0} x\right) \sinh \left(b_{0} x\right)\right. \\
& \left.+\frac{5}{3} b_{0} x-2 b_{0} x \cosh \left(b_{0} x\right)^{2}+\frac{2}{3}\left(b_{0} x\right)^{2} \cosh \left(b_{0} x\right) \sinh \left(b_{0} x\right)-\frac{2}{9}\left(b_{0} x\right)^{3}\right) \\
& -\left(\cosh \left(b_{0} x\right)-b_{0} x \sinh \left(b_{0} x\right)\right)\left(\cosh \left(b_{0} x\right)^{2}-\frac{2}{3} b_{0} x \sinh \left(b_{0} x\right) \cosh \left(b_{0} x\right)+\frac{1}{3}\left(b_{0} x\right)^{2}\right) .
\end{aligned}
$$

Proof. The calculations in the proof of Lemma 4 show that

$$
\begin{aligned}
\varphi_{1}(0)=-\varphi_{2}(0) & =1, \\
\varphi_{1}^{\prime \prime}(1)-\frac{2 b \sinh (b)}{\cosh (b)} \varphi_{1}^{\prime}(1)+b^{2} \varphi_{1}(1) & =0, \\
\varphi_{2}^{\prime \prime}(1)-\frac{2 b \sinh (b)}{\cosh (b)} \varphi_{2}^{\prime}(1)+b^{2} \varphi_{2}(1) & =\frac{4}{3} b^{2} \cosh (b)^{2}(\cosh (b)-b \sinh (b)) .
\end{aligned}
$$

According to Lemma 4, the only even solution to the differential equation in (13) satisfying $\varphi(0)=0$ is given by

$$
\varphi(x)=\beta\left(\varphi_{1}(x)+\varphi_{2}(x)\right), \quad \beta \in \mathbb{R} .
$$


As for the second boundary datum of $\varphi$ we have

$$
\varphi^{\prime \prime}(1)-\frac{2 b \sinh (b)}{\cosh (b)} \varphi^{\prime}(1)+b^{2} \varphi(1)=\beta \cdot \frac{4}{3} b^{2} \cosh (b)^{2}(\cosh (b)-b \sinh (b)) .
$$

Now, the claim follows immediately.

Lemma 5 now allows us to prove Theorem 2, i.e. we verify the existence of precisely one bifurcation point of (12) on the branch of catenoidal minimal surface solutions.

Theorem 2. For $\lambda>0$ let $u_{\lambda}(x)=\lambda \cosh \left(\frac{x}{\lambda}\right)$ denote the minimal surface solutions of (12). Let $b_{0}=1.1996786 \ldots$ be the unique positive solution of the equation $\cosh (b)=b \sinh (b), \lambda_{0}=1 / b_{0}$. Then $\left(\lambda_{0}, u_{\lambda_{0}}\right)$ is the unique bifurcation point for (12) on the branch $\lambda \mapsto\left(\lambda, u_{\lambda}\right)$.

Proof. For the abstract bifurcation result to be applied here, we refer to [CR], see also [De, Theorem 28.6]. We put

$$
\begin{aligned}
X & :=\left\{u \in C^{4}([-1,1]), u(x)=u(-x)\right\}, \\
Y & :=\left\{u \in C^{0}([-1,1]), u(x)=u(-x)\right\} \times(0, \infty) \times \mathbb{R},
\end{aligned}
$$

endowed with the obviously corresponding norms. Moreover, let

$$
\Omega:=\{u \in X, u(x)>0\} .
$$

For $v \in \Omega$ we define

$$
\begin{aligned}
L(v): & X \rightarrow Y, \\
L(v) u:= & \left(-\frac{1}{v(x) \sqrt{1+v^{\prime}(x)^{2}}} \partial_{x}\left(\frac{v(x)}{\sqrt{1+v^{\prime}(x)^{2}}} \partial_{x}\left(\frac{u^{\prime \prime}(x)}{2\left(1+v^{\prime}(x)^{2}\right)^{3 / 2}}\right)\right),\right. \\
M \quad: \quad & \left.\Omega \rightarrow Y, \quad \frac{u^{\prime \prime}(1)}{2\left(1+v^{\prime}(1)^{2}\right)^{3 / 2}}\right) \\
M(v):= & \left(\frac{1}{v(x) \sqrt{1+v^{\prime}(x)^{2}}} \partial_{x}\left(\frac{v(x)}{\sqrt{1+v^{\prime}(x)^{2}}} \partial_{x}\left(\frac{1}{2 v(x) \sqrt{1+v^{\prime}(x)^{2}}}\right)\right)\right. \\
& \left.+2 H(v)\left(H(v)^{2}-K(v)\right), 0, \frac{1}{2 v(1) \sqrt{1+v^{\prime}(1)^{2}}}\right) .
\end{aligned}
$$

We remark that it is a straightforward exercise to show that for $v \in \Omega, L(v): X \rightarrow Y$ is bijective, bounded and boundedly invertible. Moreover $L(v)^{-1}$ maps $Y \cap\left(C^{1}([-1,1]) \times(0, \infty) \times \mathbb{R}\right)$ boundedly into $X \cap C^{5}([-1,1])$. Making use of this notation, the bifurcation equation (12) becomes

$$
\begin{aligned}
0 & =L(u) u+M(u)-(0, \lambda, 0) \\
\Leftrightarrow \quad 0 & =u+L(u)^{-1}(M(u))-\lambda=: G(\lambda, u)
\end{aligned}
$$

with $G:(0, \infty) \times \Omega \rightarrow X$. In the latter formulation, thanks to the regularising properties of $L(u)^{-1}$, we gain the required compactness. Lemma 5 shows that $\left(\lambda_{0}, u_{\lambda_{0}}\right)$ is the only point on the minimal surfaces branch, where the necessary null space condition for bifurcation is satisfied.

It remains to check the transversality condition to prove that we have indeed a bifurcation point in $\left(\lambda_{0}, u_{\lambda_{0}}\right)$. Let $\varphi(x)=\varphi_{1}(x)+\varphi_{2}(x)$ be as in Lemma $5, u_{\lambda}(x)=\lambda \cosh (x / \lambda)$. Then we have to show that the equation

$$
D_{u} G\left(\lambda_{0}, u_{\lambda_{0}}\right) \psi=\left.\frac{\partial}{\partial \lambda}\left(D_{u} G\left(\lambda, u_{\lambda}\right) \varphi\right)\right|_{\lambda=\lambda_{0}}
$$


has no solution $\psi \in X$. To verify this we refer to the linearisation (13) of our differential equation around $u_{\lambda}$ as $L_{\lambda}$. To this end we define

$$
\begin{aligned}
L_{\lambda}: & X \rightarrow Y, \\
L_{\lambda} \psi(x):= & \left(\partial_{x}^{2}\left(\frac{1}{2 \cosh (x / \lambda)^{4}}\left(\cosh (x / \lambda) \psi^{\prime \prime}(x)-2 \frac{\sinh (x / \lambda)}{\lambda} \psi^{\prime}(x)+\frac{\cosh (x / \lambda)}{\lambda^{2}} \psi(x)\right)\right)\right. \\
& +\frac{1}{\lambda^{2} \cosh (x / \lambda)^{6}}\left(\cosh (x / \lambda) \psi^{\prime \prime}(x)-2 \frac{\sinh (x / \lambda)}{\lambda} \psi^{\prime}(x)+\frac{\cosh (x / \lambda)}{\lambda^{2}} \psi(x)\right), \\
& \left.\psi(0), \psi^{\prime \prime}(1)-\frac{2 \sinh (1 / \lambda)}{\lambda \cosh (1 / \lambda)} \psi^{\prime}(1)+\frac{1}{\lambda^{2}} \psi(1)\right) .
\end{aligned}
$$

To prove that (15) has no solution $\psi \in X$ we prove that

$$
L_{\lambda_{0}} \psi=\left.\frac{\partial}{\partial \lambda} L_{\lambda} \varphi\right|_{\lambda=\lambda_{0}}
$$

or, equivalently, in terms of $b=\frac{1}{\lambda}, b_{0}=\frac{1}{\lambda_{0}}$

$$
L_{1 / b_{0}} \psi=-\left.b^{2} \frac{\partial}{\partial b} L_{1 / b} \varphi\right|_{b=b_{0}}
$$

has no solution $\psi \in X$.

We assume by contradiction that a solution $\psi \in X$ to (16) does indeed exist. Using that $\cosh \left(b_{0}\right)=b_{0} \sinh \left(b_{0}\right)$ and the abbreviations

$$
\begin{aligned}
A(x) & :=\frac{1}{2 \cosh \left(b_{0} x\right)^{4}}\left(\cosh \left(b_{0} x\right) \varphi^{\prime \prime}(x)-2 b_{0} \sinh \left(b_{0} x\right) \varphi^{\prime}(x)+b_{0}^{2} \cosh \left(b_{0} x\right) \varphi(x)\right) \\
& =\frac{2 b_{0}^{2}}{3 \cosh \left(b_{0} x\right)}\left(\cosh \left(b_{0} x\right)-b_{0} x \sinh \left(b_{0} x\right)\right), \\
B(x) & :=\frac{1}{2 \cosh \left(b_{0} x\right)^{4}}\left(\cosh \left(b_{0} x\right) \psi^{\prime \prime}(x)-2 b_{0} \sinh \left(b_{0} x\right) \psi^{\prime}(x)+b_{0}^{2} \cosh \left(b_{0} x\right) \psi(x)\right), \\
C & :=\left(-2 \frac{\sinh \left(b_{0}\right)}{\cosh \left(b_{0}\right)}+2 b_{0} \frac{\sinh \left(b_{0}\right)^{2}}{\cosh \left(b_{0}\right)^{2}}-2 b_{0}\right) \varphi^{\prime}(1)+2 b_{0} \varphi(1)=-2 b_{0} \varphi^{\prime}(1)+2 b_{0} \varphi(1),
\end{aligned}
$$

this means that $\psi$ is even and solves

$$
\left\{\begin{array}{l}
B^{\prime \prime}(x)+\frac{2 b_{0}^{2}}{\cosh \left(b_{0} x\right)^{2}} B(x)=-\left.b_{0}^{2}\left(\frac{\partial}{\partial b} L_{1 / b} \varphi(x)\right)_{1}\right|_{b=b_{0}}, x \in[0,1], \\
\psi(0)=0, \\
B(1)=-\frac{b_{0}^{2} C}{2 \cosh \left(b_{0}\right)^{3}} .
\end{array}\right.
$$

Here $\left(\frac{\partial}{\partial b} L_{1 / b} \varphi(x)\right)_{1}$ denotes the first component of the triplet of $\frac{\partial}{\partial b} L_{1 / b} \varphi(x)$, i.e. the differential operator component applied to $\varphi$. We multiply the differential equation by $A(x)$ and integrate by parts:

$$
\begin{aligned}
& -\left.b_{0}^{2} \int_{0}^{1} A(x)\left(\frac{\partial}{\partial b} L_{1 / b} \varphi(x)\right)_{1}\right|_{b=b_{0}} d x \\
& =\int_{0}^{1}\left(B^{\prime \prime}(x)+\frac{2 b_{0}^{2}}{\cosh \left(b_{0} x\right)^{2}} B(x)\right) A(x) d x \\
& =\left[B^{\prime}(x) A(x)\right]_{0}^{1}-\left[B(x) A^{\prime}(x)\right]_{0}^{1}+\int_{0}^{1} B(x)\left(A^{\prime \prime}(x)+\frac{2 b_{0}^{2}}{\cosh \left(b_{0} x\right)^{2}} A(x)\right) \\
& =-B(1) A^{\prime}(1)=\frac{b_{0}^{2} C}{2 \cosh \left(b_{0}\right)^{3}} A^{\prime}(1) .
\end{aligned}
$$


In the last step we used that $A(1)=0$ and that $A$ and $B$ are even so that $A^{\prime}(0)=B^{\prime}(0)=0$. To sum up: Assuming that $\psi$ is an even solution to (17) yields the necessary condition:

$$
\left.\int_{0}^{1} A(x)\left(\frac{\partial}{\partial b} L_{1 / b} \varphi(x)\right)_{1}\right|_{b=b_{0}} d x=-\frac{C}{2 \cosh \left(b_{0}\right)^{3}} A^{\prime}(1)
$$

equivalently

$$
\left.\int_{0}^{1}\left(1-\frac{b_{0} x \sinh \left(b_{0} x\right)}{\cosh \left(b_{0} x\right)}\right)\left(\frac{\partial}{\partial b} L_{1 / b} \varphi(x)\right)_{1}\right|_{b=b_{0}} d x=\frac{C b_{0}^{2}}{2 \cosh \left(b_{0}\right)^{3}} .
$$

According to maple ${ }^{\mathrm{TM}}$ we have that

$$
\begin{aligned}
\left.\frac{\partial}{\partial b} L_{1 / b} \varphi\right|_{b=b_{0}}= & \left(-\frac{2 b_{0}^{3}}{9 \cosh \left(b_{0} x\right)^{6}}\left(-6\left(b_{0} x\right)^{2} \cosh \left(b_{0} x\right)^{4}+8\left(b_{0} x\right)^{4} \cosh \left(b_{0} x\right)^{2}\right.\right. \\
& +42\left(b_{0} x\right)^{2} \cosh \left(b_{0} x\right)^{2}+69 \cosh \left(b_{0} x\right)^{2}-18 \cosh \left(b_{0} x\right)^{6}-27 \cosh \left(b_{0} x\right)^{4} \\
& -9\left(b_{0} x\right)^{4}-54\left(b_{0} x\right)^{2}-123 b_{0} x \sinh \left(b_{0} x\right) \cosh \left(b_{0} x\right)+6 b_{0} x \cosh \left(b_{0} x\right)^{3} \sinh \left(b_{0} x\right) \\
& \left.-16\left(b_{0} x\right)^{3} \cosh \left(b_{0} x\right) \sinh \left(b_{0} x\right)\right), \\
& 0,2 b_{0}\left(-2 b_{0} \sinh \left(b_{0}\right) \cosh \left(b_{0}\right)^{2}+\frac{1}{9} b_{0}^{3} \sinh \left(b_{0}\right)-\frac{1}{9} b_{0}^{4} \cosh \left(b_{0}\right)\right. \\
& \left.\left.-\frac{5}{3} b_{0}^{2} \cosh \left(b_{0}\right)+\frac{5}{3} b_{0}^{2} \cosh \left(b_{0}\right)^{3}\right)\right) ; \\
\left.\int_{0}^{1}\left(1-\frac{b_{0} x \sinh \left(b_{0} x\right)}{\cosh \left(b_{0} x\right)}\right)\left(\frac{\partial}{\partial b} L_{1 / b} \varphi(x)\right)_{1}\right|_{b=b_{0}} d x=1.0441 \ldots ; & \frac{C b_{0}^{2}}{2 \cosh \left(b_{0}\right)^{3}}=-0.6125 \ldots
\end{aligned}
$$

With these calculations we finally obtain a contradiction with (18), so that (17) has indeed no even solution $\psi$.

Further very precise local information on the bifurcating branch is available. In the following result we calculate explicitly its full tangent vector, which can be directly compared with numerical approximations explained below in Section 4 .

Theorem 3. Let $t \mapsto\left(\lambda(t), u_{\lambda(t)}+v(t)\right)$ for $t$ close to 0 be the branch of non-minimal-surfacesolutions to (12) bifurcating from $\lambda \mapsto\left(\lambda, u_{\lambda}\right)$ in $\lambda_{0}=1 / b_{0}$ according to Theorem 2 such that $v^{\prime}(0)=$ $\varphi$, where $\varphi$ is defined in Lemma 5. Moreover, let $A(x)=\frac{2 b_{0}^{2}}{3 \cosh \left(b_{0} x\right)}\left(\cosh \left(b_{0} x\right)-b_{0} x \sinh \left(b_{0} x\right)\right)$ be as in the preceding proof. Then,

$$
\lambda^{\prime}(0)=\frac{\frac{2}{9} \int_{0}^{1} A^{3} \cosh \left(b_{0} x\right)^{2} d x+\frac{5}{9} \int_{0}^{1} A^{3} d x-\frac{2}{3} b_{0}^{2} \int_{0}^{1} \frac{\varphi A^{2}}{\cosh \left(b_{0} x\right)^{3}} d x}{\int_{0}^{1} \frac{A^{3}}{\cosh \left(b_{0} x\right)^{2}} d x}=0.8077 \ldots
$$

For the curve $t \mapsto(\alpha(t), \lambda(t))=\left(\left.\left(u_{\lambda(t)}+v(t)\right)\right|_{x=1}, \lambda(t)\right)$ as displayed in the bifurcation diagram Figure 3, in the bifurcation point we get the tangent vector

$$
\left(\alpha^{\prime}(0), \lambda^{\prime}(0)\right)=\left(\varphi(1), \lambda^{\prime}(0)\right)=(1.398 \ldots, 0.8077 \ldots)=1.398 \ldots \cdot(1,0.5776 \ldots) .
$$

For the profile of the bifurcating direction we find

$$
\left.\frac{d}{d t}\left(\lambda(t), u_{\lambda(t)}+v(t)\right)\right|_{t=0}=\left(\lambda^{\prime}(0), x \mapsto \lambda^{\prime}(0)\left(\cosh \left(b_{0} x\right)-b_{0} x \sinh \left(b_{0} x\right)\right)+\varphi(x)\right) .
$$


Proof. We denote by $t \mapsto(\lambda(t), u(t)):=\left(\lambda(t), u_{\lambda(t)}+v(t)\right)$ the bifurcating branch of non-minimalsurface-solutions to (12), where

$$
u(t)=u_{\lambda(t)}+t \varphi+o(t)=u_{\lambda_{0}}+t \eta+o(t), \quad \eta:=\lambda^{\prime}(0) \varphi_{1}+\varphi
$$

and $\varphi_{1}(x)=\cosh \left(b_{0} x\right)-b_{0} x \sinh \left(b_{0} x\right)$ is as in Lemma 5. The starting point is the differential equation satisfied by $u(t)$,

$$
\partial_{x}\left(\frac{u(t)}{\sqrt{1+u_{x}^{2}(t)}} \partial_{x} H(u(t))\right)+2 u(t) \sqrt{1+u_{x}^{2}(t)} H(u(t))\left(H(u(t))^{2}-K(u(t))\right)=0 \text { on }[-1,1] .
$$

This equation is differentiated twice with respect to $t$ in $t=0$. Writing

$$
H_{1}:=\left.\frac{\partial}{\partial t} H(u(t))\right|_{t=0}, \quad H_{2}:=\left.\frac{\partial^{2}}{\partial t^{2}} H(u(t))\right|_{t=0}, \quad K_{1}:=\left.\frac{\partial}{\partial t} K(u(t))\right|_{t=0},
$$

we find

$$
\begin{aligned}
0= & 2 \partial_{x}\left(\left(\frac{\eta}{\cosh \left(b_{0} x\right)}-\frac{\sinh \left(b_{0} x\right)}{b_{0} \cosh \left(b_{0} x\right)^{2}} \eta^{\prime}\right) H_{1}^{\prime}\right)+\frac{1}{b_{0}} H_{2}^{\prime \prime}+\frac{2 b_{0}}{\cosh \left(b_{0} x\right)^{2}} H_{2} \\
& -\frac{4}{b_{0}} \cosh \left(b_{0} x\right)^{2} K_{1} H_{1}+\frac{4 b_{0}^{2}}{\cosh \left(b_{0} x\right)^{4}}\left(\eta \cosh \left(b_{0} x\right)+\frac{1}{b_{0}} \eta^{\prime} \sinh \left(b_{0} x\right)\right) H_{1} .
\end{aligned}
$$

One calculates

$$
\begin{aligned}
& H_{1}=D_{u} H\left(u_{\lambda_{0}}\right) \eta=D_{u} H\left(u_{\lambda_{0}}\right) \varphi=-A \\
& K_{1}=-\frac{b_{0}}{\cosh \left(b_{0} x\right)^{5}} \eta^{\prime \prime}+4 \frac{b_{0}^{2} \sinh \left(b_{0} x\right)}{\cosh \left(b_{0} x\right)^{6}} \eta^{\prime}+\frac{b_{0}^{3}}{\cosh \left(b_{0} x\right)^{5}} \eta .
\end{aligned}
$$

Observing that $\eta=\lambda^{\prime}(0) \varphi_{1}+\varphi$, after some lengthy and tedious calculations and making use of the differential equation (14) satisfied by $\varphi$ and of $A^{\prime \prime}+\frac{2 b_{0}^{2}}{\cosh \left(b_{0} x\right)^{2}} A=0$, we come up with

$$
\begin{aligned}
0= & \frac{4}{b_{0}} \sinh \left(b_{0} x\right) \cosh \left(b_{0} x\right) A A^{\prime}+\frac{1}{b_{0}}\left(H_{2}^{\prime \prime}+\frac{2 b_{0}^{2}}{\cosh \left(b_{0} x\right)^{2}} H_{2}\right) \\
& +12 \frac{A^{2}}{\cosh \left(b_{0} x\right)^{2}} \lambda^{\prime}(0)+8 \frac{b_{0}^{2}}{\cosh \left(b_{0} x\right)^{3}} \varphi A-8 A^{2} .
\end{aligned}
$$

This identity is now multiplied by $A$ and integrated on $(0,1)$. The term containing $H_{2}$ is shown to vanish by integrating by parts. Since $H_{2}$ and $A$ are even in $x$, we have $H_{2}^{\prime}(0)=A^{\prime}(0)=0$. Since $H(u(t))(1)=1$, we also have $H_{2}(1)=0$ as well as $A(1)=0$. Exploiting $A^{\prime \prime}+\frac{2 b_{0}^{2}}{\cosh \left(b_{0} x\right)^{2}} A=0$ again then yields formula (19). The proof of the remaining claims is then straightforward.

Remark 2. For $\alpha$ close to $\alpha_{0}$ we can think of the branch of non-minimal surface solutions as being parametrised by $\alpha$, say $\alpha \mapsto u_{\alpha},\left|\alpha-\alpha_{0}\right|<\varepsilon$. It then follows from Theorem 3 that $\left.\frac{\partial u_{\alpha}}{\partial \alpha}\right|_{\alpha=\alpha_{0}}=w$, where

$$
w(x)=\frac{1}{\varphi(1)}\left(\lambda^{\prime}(0)\left(\cosh \left(b_{0} x\right)-b_{0} x \sinh \left(b_{0} x\right)\right)+\varphi(x)\right) .
$$

This profile as well as its numerically calculated counterpart, which is obtained from the numerical approximations on the branch of "nontrivial" solutions, is displayed in Figure 5. 


\section{Numerical approximation}

In this section we present a numerical method which allows the calculation of approximate solutions of (4) irrespective of whether the solution corresponds to a local minimum or a saddle point of the Willmore functional. As we shall use a finite element approach, the first step consists in deriving a suitable variational form of the differential equation in (4). To begin, we introduce the new variable

$$
w(x):=H(x) \sqrt{1+u^{\prime}(x)^{2}} .
$$

Let us express the operator $\Delta_{\mathcal{R}} H+2 H\left(H^{2}-K\right)$ in terms $u$ and $w$. Note that

$$
H^{\prime}=\frac{w^{\prime}}{\sqrt{1+\left(u^{\prime}\right)^{2}}}-\frac{u^{\prime} u^{\prime \prime} H}{1+\left(u^{\prime}\right)^{2}} .
$$

Recalling (6) and that $H=-\frac{u^{\prime \prime}}{2\left(1+\left(u^{\prime}\right)^{2}\right)^{3 / 2}}+\frac{1}{2 u \sqrt{1+\left(u^{\prime}\right)^{2}}}$ we have

$$
\begin{aligned}
u \Delta_{\mathcal{R}} H & =\frac{1}{\sqrt{1+\left(u^{\prime}\right)^{2}}} \partial_{x}\left(\frac{u}{\sqrt{1+\left(u^{\prime}\right)^{2}}} H^{\prime}\right) \\
& =\partial_{x}\left(\frac{u}{1+\left(u^{\prime}\right)^{2}} H^{\prime}\right)+\frac{u u^{\prime} u^{\prime \prime} H^{\prime}}{\left(1+\left(u^{\prime}\right)^{2}\right)^{2}} \\
& =\partial_{x}\left(\frac{u w^{\prime}}{\left(1+\left(u^{\prime}\right)^{2}\right)^{3 / 2}}\right)-\partial_{x}\left(\frac{u u^{\prime} u^{\prime \prime} H}{\left(1+\left(u^{\prime}\right)^{2}\right)^{2}}\right)+\frac{u u^{\prime} u^{\prime \prime} H^{\prime}}{\left(1+\left(u^{\prime}\right)^{2}\right)^{2}} \\
& =\partial_{x}\left(\frac{u w^{\prime}}{\left(1+\left(u^{\prime}\right)^{2}\right)^{3 / 2}}\right)-H \partial_{x}\left(\frac{u u^{\prime} u^{\prime \prime}}{\left(1+\left(u^{\prime}\right)^{2}\right)^{2}}\right) \\
& =\partial_{x}\left(\frac{u w^{\prime}}{\left(1+\left(u^{\prime}\right)^{2}\right)^{3 / 2}}\right)+2 H \partial_{x}\left(\frac{u u^{\prime} H}{\sqrt{1+\left(u^{\prime}\right)^{2}}}\right)-H \partial_{x}\left(\frac{u^{\prime}}{1+\left(u^{\prime}\right)^{2}}\right)
\end{aligned}
$$

We calculate for the second term

$$
\begin{aligned}
2 H \partial_{x}\left(\frac{u u^{\prime} H}{\sqrt{1+\left(u^{\prime}\right)^{2}}}\right)=2 H H^{\prime} \frac{u u^{\prime}}{\sqrt{1+\left(u^{\prime}\right)^{2}}}+2 H^{2} \partial_{x}\left(\frac{u u^{\prime}}{\sqrt{1+\left(u^{\prime}\right)^{2}}}\right) \\
\quad=\partial_{x}\left(\frac{u u^{\prime} H^{2}}{\sqrt{1+\left(u^{\prime}\right)^{2}}}\right)+H^{2} \partial_{x}\left(\frac{u u^{\prime}}{\sqrt{1+\left(u^{\prime}\right)^{2}}}\right) \\
\quad=\partial_{x}\left(\frac{u u^{\prime} w^{2}}{\left(1+\left(u^{\prime}\right)^{2}\right)^{3 / 2}}\right)+H^{2}\left(\frac{\left(u^{\prime}\right)^{2}}{\sqrt{1+\left(u^{\prime}\right)^{2}}}+u \frac{u^{\prime \prime}}{\left(1+\left(u^{\prime}\right)^{2}\right)^{3 / 2}}\right) \\
=\partial_{x}\left(\frac{u u^{\prime} w^{2}}{\left(1+\left(u^{\prime}\right)^{2}\right)^{3 / 2}}\right)+H^{2}\left\{\frac{\left(u^{\prime}\right)^{2}}{\sqrt{1+\left(u^{\prime}\right)^{2}}}+u\left(-2 H+\frac{1}{u \sqrt{1+\left(u^{\prime}\right)^{2}}}\right)\right\} \\
=\partial_{x}\left(\frac{u u^{\prime} w^{2}}{\left(1+\left(u^{\prime}\right)^{2}\right)^{3 / 2}}\right)+H^{2} \sqrt{1+\left(u^{\prime}\right)^{2}}-2 u H^{3} .
\end{aligned}
$$

On the other hand, using (5)

$$
\partial_{x}\left(\frac{u^{\prime}}{1+\left(u^{\prime}\right)^{2}}\right)=-\frac{u^{\prime \prime}}{1+\left(u^{\prime}\right)^{2}}+2 \frac{u^{\prime \prime}}{\left(1+\left(u^{\prime}\right)^{2}\right)^{2}}=2 H \sqrt{1+\left(u^{\prime}\right)^{2}}-\frac{1}{u}-2 u K .
$$

Inserting the two identities into (23) above we obtain

$$
\begin{aligned}
& u\left(\Delta_{\mathcal{R}} H+2 H\left(H^{2}-K\right)\right) \\
& \quad=\partial_{x}\left(\frac{u w^{\prime}}{\left(1+\left(u^{\prime}\right)^{2}\right)^{3 / 2}}\right)+\partial_{x}\left(\frac{u u^{\prime} w^{2}}{\left(1+\left(u^{\prime}\right)^{2}\right)^{3 / 2}}\right)+\frac{w}{\sqrt{1+\left(u^{\prime}\right)^{2}}}\left(\frac{1}{u}-w\right) .
\end{aligned}
$$


Thus, the equation $\Delta_{\mathcal{R}} H+2 H\left(H^{2}-K\right)=0$ translates into

$$
\partial_{x}\left(\frac{u w^{\prime}}{\left(1+\left(u^{\prime}\right)^{2}\right)^{3 / 2}}\right)+\partial_{x}\left(\frac{u u^{\prime} w^{2}}{\left(1+\left(u^{\prime}\right)^{2}\right)^{3 / 2}}\right)+\frac{w}{\sqrt{1+\left(u^{\prime}\right)^{2}}}\left(\frac{1}{u}-w\right)=0, \quad \text { in }(-1,1),
$$

which can be written in variational form as

$$
\int_{-1}^{1} \frac{u w^{\prime} \phi^{\prime}}{\left(1+\left(u^{\prime}\right)^{2}\right)^{3 / 2}}+\int_{-1}^{1} \frac{u u^{\prime} w^{2} \phi^{\prime}}{\left(1+\left(u^{\prime}\right)^{2}\right)^{3 / 2}}-\int_{-1}^{1} \frac{w \phi}{\sqrt{1+\left(u^{\prime}\right)^{2}}}\left(\frac{1}{u}-w\right)=0
$$

for all $\phi \in H_{0}^{1}(-1,1)$. In order to establish the relation between $u$ and $w$ we observe that

$$
\begin{aligned}
\partial_{x}\left(\frac{u u^{\prime}}{\sqrt{1+\left(u^{\prime}\right)^{2}}}\right) & =\frac{\left(u^{\prime}\right)^{2}}{\sqrt{1+\left(u^{\prime}\right)^{2}}}+\frac{u u^{\prime \prime}}{\left(1+\left(u^{\prime}\right)^{2}\right)^{3 / 2}} \\
& =\frac{\left(u^{\prime}\right)^{2}}{\sqrt{1+\left(u^{\prime}\right)^{2}}}+u\left(-2 H+\frac{1}{u \sqrt{1+\left(u^{\prime}\right)^{2}}}\right) \\
& =-2 u H+\sqrt{1+\left(u^{\prime}\right)^{2}}=-2 \frac{u w}{\sqrt{1+\left(u^{\prime}\right)^{2}}}+\sqrt{1+\left(u^{\prime}\right)^{2}}
\end{aligned}
$$

which, in variational form, reads

$$
2 \int_{-1}^{1} \frac{u w \eta}{\sqrt{1+\left(u^{\prime}\right)^{2}}}-\int_{-1}^{1} \frac{u u^{\prime} \eta^{\prime}}{\sqrt{1+\left(u^{\prime}\right)^{2}}}-\int_{-1}^{1} \sqrt{1+\left(u^{\prime}\right)^{2}} \eta=0 \quad \forall \eta \in H_{0}^{1}(-1,1) .
$$

In addition to (24) and (25) we impose the boundary conditions

$$
u( \pm 1)=\alpha, \quad w( \pm 1)=0 .
$$

Since (24), (25) only contains first order derivatives it is natural to approximate $u$ and $w$ by piecewise linear continuous functions. Thus, let $x_{j}=-1+j h, j=0, \ldots, N+1, h=\frac{2}{N+1}$ and define

$$
X_{h}:=\left\{\phi_{h} \in C^{0}([-1,1]) \mid \phi_{h \mid\left[x_{j-1}, x_{j}\right]} \in P_{1}, j=1, \ldots, N+1\right\},
$$

where $P_{1}$ is the space of polynomials of degree less than or equal to 1 . Furthermore, we set $X_{h 0}:=X_{h} \cap H_{0}^{1}(-1,1)$.

We now propose the following scheme: find $u_{h} \in X_{h}, u_{h}( \pm 1)=\alpha, w_{h} \in X_{h 0}$ such that $u_{h}>0$ in $[-1,1]$ and for all $\phi_{h}, \eta_{h} \in X_{h 0}$

$$
\begin{aligned}
& \int_{-1}^{1} \frac{u_{h} w_{h}^{\prime} \phi_{h}^{\prime}}{\left(1+\left(u_{h}^{\prime}\right)^{2}\right)^{3 / 2}}+\int_{-1}^{1} \frac{u_{h} u_{h}^{\prime} w_{h}^{2} \phi_{h}^{\prime}}{\left(1+\left(u_{h}^{\prime}\right)^{2}\right)^{3 / 2}}-\int_{-1}^{1} \frac{w_{h} \phi_{h}}{\sqrt{1+\left(u_{h}^{\prime}\right)^{2}}}\left(\frac{1}{u_{h}}-w_{h}\right)=0 \\
& 2 \int_{-1}^{1} \frac{u_{h} w_{h} \eta_{h}}{\sqrt{1+\left(u_{h}^{\prime}\right)^{2}}}-\int_{-1}^{1} \frac{u_{h} u_{h}^{\prime} \eta_{h}^{\prime}}{\sqrt{1+\left(u_{h}^{\prime}\right)^{2}}}-\int_{-1}^{1} \sqrt{1+\left(u_{h}^{\prime}\right)^{2}} \eta_{h}=0 .
\end{aligned}
$$

Note that the hat functions $\phi_{j} \in X_{h}$ with $\phi_{j}\left(x_{k}\right)=\delta_{j k}, j, k=0,1, \ldots, N+1$ form a basis of $X_{h}$, while $\phi_{1}, \ldots, \phi_{N}$ are a basis of $X_{h 0}$. We define $\Omega:=\left\{(\underline{u}, \underline{w}) \in \mathbb{R}^{2 N} \mid u_{i}>0, i=1, \ldots, N\right\}$ and assign to each $(\underline{u}, \underline{w}) \in \Omega, \underline{u}=\left(u_{1}, \ldots, u_{N}\right), \underline{w}=\left(w_{1}, \ldots, w_{N}\right)$ the functions

$$
u_{h}=\alpha \phi_{0}+\sum_{i=1}^{N} u_{i} \phi_{i}+\alpha \phi_{N+1} \in X_{h}, \quad w_{h}=\sum_{i=1}^{N} w_{i} \phi_{i} \in X_{h 0} .
$$


This allows us to translate (26), (27) into a system of nonlinear equations for the coefficients $u_{1}, \ldots, u_{N}, w_{1}, \ldots, w_{N}$. To do so, define $F: \Omega \rightarrow \mathbb{R}^{2 N}$ by

$$
\begin{aligned}
F_{j}(\underline{u}, \underline{w}) & :=\int_{-1}^{1} \frac{u_{h} w_{h}^{\prime} \phi_{j}^{\prime}}{\left(1+\left(u_{h}^{\prime}\right)^{2}\right)^{3 / 2}}+\int_{-1}^{1} \frac{u_{h} u_{h}^{\prime} w_{h}^{2} \phi_{j}^{\prime}}{\left(1+\left(u_{h}^{\prime}\right)^{2}\right)^{3 / 2}}-\int_{-1}^{1} \frac{w_{h} \phi_{j}}{\sqrt{1+\left(u_{h}^{\prime}\right)^{2}}}\left(\frac{1}{u_{h}}-w_{h}\right), \\
F_{j+N}(\underline{u}, \underline{w}) & :=2 \int_{-1}^{1} \frac{u_{h} w_{h} \phi_{j}}{\sqrt{1+\left(u_{h}^{\prime}\right)^{2}}}-\int_{-1}^{1} \frac{u_{h} u_{h}^{\prime} \phi_{j}^{\prime}}{\sqrt{1+\left(u_{h}^{\prime}\right)^{2}}}-\int_{-1}^{1} \sqrt{1+\left(u_{h}^{\prime}\right)^{2}} \phi_{j},
\end{aligned}
$$

for $1 \leq j \leq N$. It is straightforward to see that the scheme $(26),(27)$ is equivalent to the solution of the system

$$
F(\underline{u}, \underline{w})=0 .
$$

We used Newton's method in order to obtain approximate solutions of (28). To start the iteration we picked a vector $\underline{u}^{(0)}$ with $u_{i}^{(0)}>0,1 \leq i \leq N$ and then calculated the vector $\underline{w}^{(0)}$ via the finite difference formula (cf. (22))

$$
\begin{aligned}
w_{i}^{(0)}:= & \frac{1}{2}\left(\sqrt{1+\left(\delta^{+} u_{i}^{(0)}\right)^{2}}+\sqrt{1+\left(\delta^{-} u_{i}^{(0)}\right)^{2}}\right)\left\{\frac{-1}{2 h}\left(\frac{\delta^{+} u_{i}^{(0)}}{\sqrt{1+\left(\delta^{+} u_{i}^{(0)}\right)^{2}}}-\frac{\delta^{-} u_{i}^{(0)}}{\sqrt{1+\left(\delta^{-} u_{i}^{(0)}\right)^{2}}}\right)\right. \\
& \left.+\frac{1}{4 u_{i}^{(0)}}\left(\frac{1}{\sqrt{1+\left(\delta^{+} u_{i}^{(0)}\right)^{2}}}+\frac{1}{\sqrt{1+\left(\delta^{-} u_{i}^{(0)}\right)^{2}}}\right)\right\}, \quad 1 \leq i \leq N,
\end{aligned}
$$

where

$$
\delta^{+} u_{i}^{(0)}=\frac{u_{i+1}^{(0)}-u_{i}^{(0)}}{h}, \quad \delta^{-} u_{i}^{(0)}=\frac{u_{i}^{(0)}-u_{i-1}^{(0)}}{h} .
$$

The choice of $\underline{u}^{(0)}$ was determined by the value of $\alpha$ : In the case $0<\alpha<\alpha_{0}$ we simply took

$$
u_{i}^{(0)}:=\alpha, \quad 1 \leq i \leq N
$$

and observed convergence of Newton's method, at least as long as $\alpha \geq 0.05$. We denote this solution by $u_{h, \alpha}$.

For $\alpha>\alpha_{0}$ we chose

$$
u_{i}^{(0)}=\lambda \frac{\cosh \left(b_{\alpha} x_{i}\right)}{b_{\alpha}}+(1-\lambda) \frac{\cosh \left(b_{\alpha}^{\prime} x_{i}\right)}{b_{\alpha}^{\prime}}, \quad 1 \leq i \leq N,
$$

where $\lambda \in[0,1]$ and $b_{\alpha}, b_{\alpha}^{\prime}$ are the two (approximate) solutions of the equation $\cosh (b) / b=\alpha$. If we chose $\lambda$ close to either 1 or 0 we observed convergence towards the minimal surface solutions $x \mapsto \cosh \left(b_{\alpha} x\right) / b_{\alpha}$ and $x \mapsto \cosh \left(b_{\alpha}^{\prime} x\right) / b_{\alpha}^{\prime}$ respectively. For the choice $\lambda=\frac{1}{2}$ Newton's method converged to a third solution which we denote by $u_{h, \alpha}$ and which we expected to lie (approximately) on the branch of non-minimal surface solutions. Figure 4 shows an example of a calculation for $\alpha=1.6$. The graph of the non-minimal surface solution is displayed together with those of the two catenoid solutions and lies between them.

In order to verify that the functions $u_{h, \alpha}$ lie indeed on the nontrivial branch we evaluated the expression

$$
w_{h}(x)=\frac{1}{12 \delta}\left(u_{h, \alpha_{0}-2 \delta}(x)-8 u_{h, \alpha_{0}-\delta}(x)+8 u_{h, \alpha_{0}+\delta}(x)-u_{h, \alpha_{0}+2 \delta}(x)\right)
$$

and calculated an approximation to the derivative of $\alpha \mapsto u_{h, \alpha}$ with respect to $\alpha$. The resulting function is shown in Fig. 5 (right) for the choices $h=0.0025$ and $\delta=0.1$ and is in very good agreement with the function $w$ in Remark 2 . 


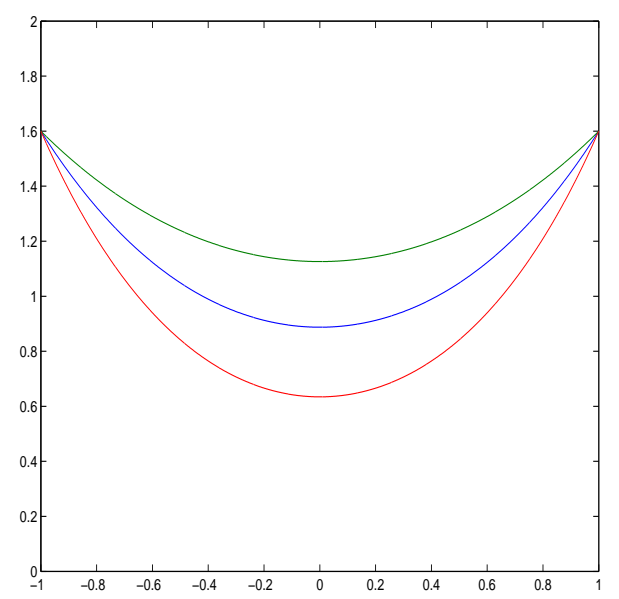

Figure 4: Numerically calculated non-minimal surface solution and the two catenoid solutions for $\alpha=1.6$.

\section{A First and second variation of $W$}

The aim of this section is to derive, for the reader's convenience, formulae for the first and second variation of the Willmore functional

$$
\tilde{W}(u):=\frac{1}{\pi} W(u)=\frac{1}{2} \int_{-1}^{1}\left(\frac{1}{u(x) \sqrt{1+u^{\prime}(x)^{2}}}-\frac{u^{\prime \prime}(x)}{\left(1+u^{\prime}(x)^{2}\right)^{3 / 2}}\right)^{2} u(x) \sqrt{1+u^{\prime}(x)^{2}} d x .
$$

We recall that the corresponding Euler-Lagrange is well known. However, since we are in a quite symmetric setting, the formulae and calculations become particularly simple. The above expression can be rewritten in a slightly more convenient way:

$$
\tilde{W}(u)=\frac{1}{2} \int_{-1}^{1} \frac{1}{u(x) \sqrt{1+u^{\prime}(x)^{2}}} d x+\frac{1}{2} \int_{-1}^{1} \frac{u(x) u^{\prime \prime}(x)^{2}}{\left(1+u^{\prime}(x)^{2}\right)^{5 / 2}} d x-\left[\frac{u^{\prime}(x)}{\sqrt{1+u^{\prime}(x)^{2}}}\right]_{-1}^{1} .
$$

Lemma 6. Let $u \in C^{4}([-1,1],(0, \infty))$ and $\varphi, \eta \in H^{2}(-1,1) \cap H_{0}^{1}(-1,1)$. Then

$$
\begin{aligned}
\left\langle\tilde{W}^{\prime}(u), \varphi\right\rangle= & -2\left[H \frac{u \varphi^{\prime}}{1+\left(u^{\prime}\right)^{2}}\right]_{x=-1}^{x=1}-2 \int_{-1}^{1} u \varphi\left(\Delta_{\mathcal{R}} H+2 H\left(H^{2}-K\right)\right) d x \\
\tilde{W}^{\prime \prime}(u)(\varphi, \eta)= & \int_{-1}^{1}\left(\frac{\varphi \eta}{u^{3}\left(1+\left(u^{\prime}\right)^{2}\right)^{1 / 2}}+\frac{u^{\prime}(\varphi \eta)^{\prime}}{2 u^{2}\left(1+\left(u^{\prime}\right)^{2}\right)^{3 / 2}}\right) d x \\
& +\int_{-1}^{1}\left(\frac{-\varphi^{\prime} \eta^{\prime}}{2 u\left(1+\left(u^{\prime}\right)^{2}\right)^{3 / 2}}+\frac{3\left(u^{\prime}\right)^{2} \varphi^{\prime} \eta^{\prime}}{2 u\left(1+\left(u^{\prime}\right)^{2}\right)^{5 / 2}}\right) d x \\
& +\int_{-1}^{1}\left(\frac{u^{\prime \prime}(\varphi \eta)^{\prime \prime}}{\left(1+\left(u^{\prime}\right)^{2}\right)^{5 / 2}}-\frac{2 u^{\prime \prime} \varphi^{\prime} \eta^{\prime}}{\left(1+\left(u^{\prime}\right)^{2}\right)^{5 / 2}}-\frac{5 u^{\prime}\left(u^{\prime \prime}\right)^{2}(\varphi \eta)^{\prime}}{2\left(1+\left(u^{\prime}\right)^{2}\right)^{7 / 2}}\right) d x \\
& +\int_{-1}^{1}\left(\frac{u \varphi^{\prime \prime} \eta^{\prime \prime}}{\left(1+\left(u^{\prime}\right)^{2}\right)^{5 / 2}}-\frac{5 u u^{\prime} u^{\prime \prime}\left(\varphi^{\prime} \eta^{\prime}\right)^{\prime}}{\left(1+\left(u^{\prime}\right)^{2}\right)^{7 / 2}}\right) d x \\
& +\int_{-1}^{1}\left(\frac{-5 u\left(u^{\prime \prime}\right)^{2} \varphi^{\prime} \eta^{\prime}}{2\left(1+\left(u^{\prime}\right)^{2}\right)^{7 / 2}}+\frac{35 u\left(u^{\prime}\right)^{2}\left(u^{\prime \prime}\right)^{2} \varphi^{\prime} \eta^{\prime}}{2\left(1+\left(u^{\prime}\right)^{2}\right)^{9 / 2}}\right) d x+\left[\frac{3 u^{\prime} \varphi^{\prime} \eta^{\prime}}{\left(1+\left(u^{\prime}\right)^{2}\right)^{5 / 2}}\right]_{x=-1}^{x=1}
\end{aligned}
$$



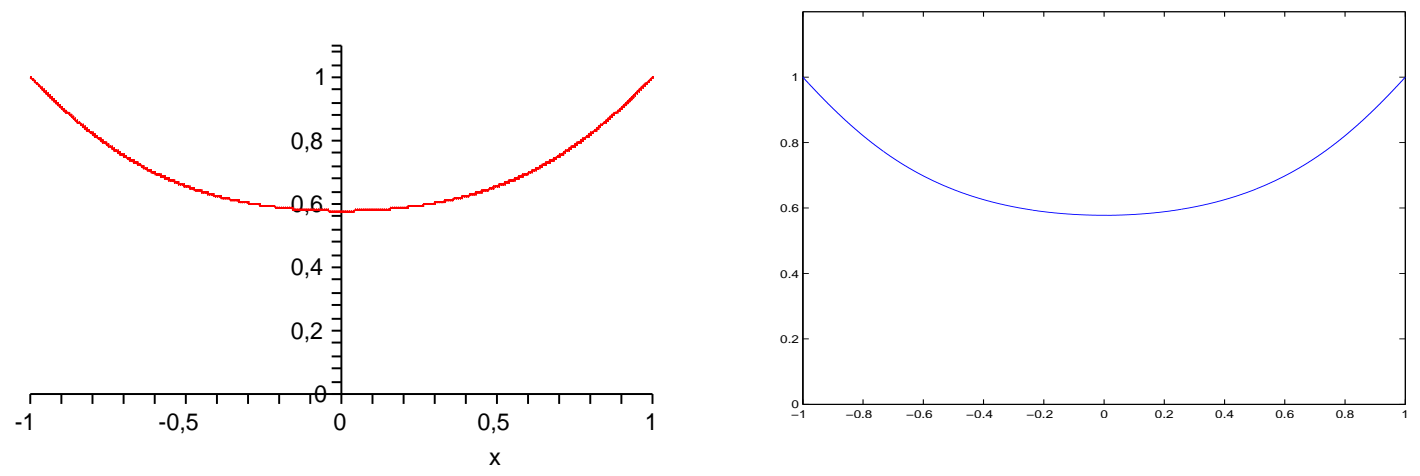

Figure 5: Profile of $w=\frac{1}{\varphi(1)}\left(\left.\lambda^{\prime}(0) \partial_{\lambda} u_{\lambda}\right|_{\lambda=\lambda_{0}}+\varphi\right)$ of the bifurcating perturbations from the branch of minimal surface solutions (left). On the right, plot of its numerical approximation $w_{h}$.

Proof. Clearly,

$$
\begin{aligned}
& \left\langle\tilde{W}^{\prime}(u), \varphi\right\rangle=\int_{-1}^{1} \frac{u u^{\prime \prime} \varphi^{\prime \prime}}{\left(1+\left(u^{\prime}\right)^{2}\right)^{\frac{5}{2}}}-\frac{5}{2} \int_{-1}^{1} \frac{u u^{\prime}\left(u^{\prime \prime}\right)^{2} \varphi^{\prime}}{\left(1+\left(u^{\prime}\right)^{2}\right)^{\frac{7}{2}}}-\frac{1}{2} \int_{-1}^{1} \frac{u^{\prime} \varphi^{\prime}}{u\left(1+\left(u^{\prime}\right)^{2}\right)^{3 / 2}} \\
& +\frac{1}{2} \int_{-1}^{1}\left(\frac{\left(u^{\prime \prime}\right)^{2} \varphi}{\left(1+\left(u^{\prime}\right)^{2}\right)^{\frac{5}{2}}}-\frac{\varphi}{u^{2}\left(1+\left(u^{\prime}\right)^{2}\right)^{\frac{1}{2}}}\right)-\left[\frac{\varphi^{\prime}}{\left(1+\left(u^{\prime}\right)^{2}\right)^{3 / 2}}\right]_{x=-1}^{x=1} \equiv I+I I+I I I+I V+V .
\end{aligned}
$$

In view of (5) we may write

$$
\frac{u^{\prime \prime}}{\left(1+\left(u^{\prime}\right)^{2}\right)^{3 / 2}}=-2 H+\frac{1}{u\left(1+\left(u^{\prime}\right)^{2}\right)^{\frac{1}{2}}} .
$$

Using (30) as well as integration by parts we derive for the first term

$$
\begin{aligned}
I= & -2 \int_{-1}^{1} \frac{u H \varphi^{\prime \prime}}{1+\left(u^{\prime}\right)^{2}}+\int_{-1}^{1} \frac{\varphi^{\prime \prime}}{\left(1+\left(u^{\prime}\right)^{2}\right)^{3 / 2}} \\
= & {\left[-2 H \frac{u \varphi^{\prime}}{1+\left(u^{\prime}\right)^{2}}+\frac{\varphi^{\prime}}{\left(1+\left(u^{\prime}\right)^{2}\right)^{3 / 2}}\right]_{-1}^{1}+2 \int_{-1}^{1} \frac{u H^{\prime} \varphi^{\prime}}{1+\left(u^{\prime}\right)^{2}}+2 \int_{-1}^{1} \frac{u^{\prime} H \varphi^{\prime}}{1+\left(u^{\prime}\right)^{2}} } \\
& -4 \int_{-1}^{1} \frac{u u^{\prime} u^{\prime \prime} H \varphi^{\prime}}{\left(1+\left(u^{\prime}\right)^{2}\right)^{2}}+3 \int_{-1}^{1} \frac{u^{\prime} u^{\prime \prime} \varphi^{\prime}}{\left(1+\left(u^{\prime}\right)^{2}\right)^{\frac{5}{2}}} \\
= & {\left[-2 H \frac{u \varphi^{\prime}}{1+\left(u^{\prime}\right)^{2}}+\frac{\varphi^{\prime}}{\left(1+\left(u^{\prime}\right)^{2}\right)^{3 / 2}}\right]_{-1}^{1}-2 \int_{-1}^{1}\left(\frac{u}{\sqrt{1+\left(u^{\prime}\right)^{2}} H^{\prime}}\right)^{\prime} \frac{\varphi}{\sqrt{1+\left(u^{\prime}\right)^{2}}}+2 \int_{-1}^{1} \frac{u^{\prime} H^{\prime} \varphi}{1+\left(u^{\prime}\right)^{2}} } \\
& -4 \int_{-1}^{1} \frac{u u^{\prime} H H^{\prime} \varphi}{\left(1+\left(u^{\prime}\right)^{2}\right)^{\frac{1}{2}}}-8 \int_{-1}^{1} \frac{u^{\prime} H \varphi^{\prime}}{1+\left(u^{\prime}\right)^{2}}+8 \int_{-1}^{1} \frac{u u^{\prime} H^{2} \varphi^{\prime}}{\left(1+\left(u^{\prime}\right)^{2}\right)^{\frac{1}{2}}}+3 \int_{-1}^{1} \frac{u^{\prime} \varphi^{\prime}}{u\left(1+\left(u^{\prime}\right)^{2}\right)^{3 / 2}} .
\end{aligned}
$$

Next, taking the square of (30) we deduce

$$
\frac{\left(u^{\prime \prime}\right)^{2}}{\left(1+\left(u^{\prime}\right)^{2}\right)^{3}}=4 H^{2}-\frac{4 H}{u\left(1+\left(u^{\prime}\right)^{2}\right)^{\frac{1}{2}}}+\frac{1}{u^{2}\left(1+\left(u^{\prime}\right)^{2}\right)} .
$$


As a consequence,

$$
I I+V=-\left[\frac{\varphi^{\prime}}{\left(1+\left(u^{\prime}\right)^{2}\right)^{3 / 2}}\right]_{-1}^{1}-10 \int_{-1}^{1} \frac{u u^{\prime} H^{2} \varphi^{\prime}}{\left(1+\left(u^{\prime}\right)^{2}\right)^{\frac{1}{2}}}+10 \int_{-1}^{1} \frac{u^{\prime} H \varphi^{\prime}}{1+\left(u^{\prime}\right)^{2}}-\frac{5}{2} \int_{-1}^{1} \frac{u^{\prime} \varphi^{\prime}}{u\left(1+\left(u^{\prime}\right)^{2}\right)^{3 / 2}}
$$

as well as

$$
I V=2 \int_{-1}^{1} H^{2} \varphi \sqrt{1+\left(u^{\prime}\right)^{2}}-2 \int_{-1}^{1} \frac{H \varphi}{u} .
$$

Thus,

$$
\begin{aligned}
I+I I+I I I+V & =-2\left[H \frac{u \varphi^{\prime}}{1+\left(u^{\prime}\right)^{2}}\right]_{-1}^{1}-2 \int_{-1}^{1} u \varphi \Delta_{\mathcal{R}} H-2 \int_{-1}^{1} \frac{u u^{\prime}\left(H^{2} \varphi\right)^{\prime}}{\left(1+\left(u^{\prime}\right)^{2}\right)^{\frac{1}{2}}}+2 \int_{-1}^{1} \frac{u^{\prime}(H \varphi)^{\prime}}{1+\left(u^{\prime}\right)^{2}} \\
= & -2\left[H \frac{u \varphi^{\prime}}{1+\left(u^{\prime}\right)^{2}}\right]_{-1}^{1}-2 \int_{-1}^{1} u \varphi \Delta_{\mathcal{R}} H+2 \int_{-1}^{1} \frac{\left(u^{\prime}\right)^{2} H^{2} \varphi}{\left(1+\left(u^{\prime}\right)^{2}\right)^{\frac{1}{2}}} \\
& +2 \int_{-1}^{1} \frac{u u^{\prime \prime} H^{2} \varphi}{\left(1+\left(u^{\prime}\right)^{2}\right)^{3 / 2}}-2 \int_{-1}^{1} \frac{u^{\prime \prime} H \varphi}{1+\left(u^{\prime}\right)^{2}}+4 \int_{-1}^{1} \frac{\left(u^{\prime}\right)^{2} u^{\prime \prime} H \varphi}{\left(1+\left(u^{\prime}\right)^{2}\right)^{2}} \\
= & -2\left[H \frac{u \varphi^{\prime}}{1+\left(u^{\prime}\right)^{2}}\right]_{-1}^{1}-2 \int_{-1}^{1} u \varphi \Delta_{\mathcal{R} H+2} \int_{-1}^{1} \frac{\left(u^{\prime}\right)^{2} H^{2} \varphi}{\left(1+\left(u^{\prime}\right)^{2}\right)^{\frac{1}{2}}}-4 \int_{-1}^{1} u \varphi H^{3} \\
& +2 \int_{-1}^{1} \frac{H^{2} \varphi}{\left(1+\left(u^{\prime}\right)^{2}\right)^{\frac{1}{2}}}-4 \int_{-1}^{1} \frac{u^{\prime \prime} H \varphi}{\left(1+\left(u^{\prime}\right)^{2}\right)^{2}}+2 \int_{-1}^{1} \frac{u^{\prime \prime} H \varphi}{1+\left(u^{\prime}\right)^{2}} .
\end{aligned}
$$

Using once more (30) and recalling the formula for $K$ we finally have

$$
\begin{aligned}
I+I I+I I I+V= & -2\left[H \frac{u \varphi^{\prime}}{1+\left(u^{\prime}\right)^{2}}\right]_{-1}^{1}-2 \int_{-1}^{1} u \varphi\left(\Delta_{\mathcal{R}} H+2 H^{3}-2 H K\right) \\
& -2 \int_{-1}^{1} H^{2} \varphi \sqrt{1+\left(u^{\prime}\right)^{2}}+2 \int_{-1}^{1} \frac{H \varphi}{u} \\
= & -2\left[H \frac{u \varphi^{\prime}}{1+\left(u^{\prime}\right)^{2}}\right]_{-1}^{1}-2 \int_{-1}^{1} u \varphi\left(\Delta_{\mathcal{R}} H+2 H^{3}-2 H K\right)-I V .
\end{aligned}
$$

In order to obtain the formula for the second variation we return to (29):

$$
\begin{aligned}
\tilde{W}^{\prime \prime}(u)(\varphi, \eta)= & \left.\frac{d}{d \varepsilon}\left\langle\tilde{W}^{\prime}(u+\varepsilon \eta), \varphi\right\rangle\right|_{\varepsilon=0} \\
= & \int_{-1}^{1}\left(\frac{u^{\prime \prime} \varphi^{\prime \prime} \eta}{\left(1+\left(u^{\prime}\right)^{2}\right)^{5 / 2}}+\frac{u \varphi^{\prime \prime} \eta^{\prime \prime}}{\left(1+\left(u^{\prime}\right)^{2}\right)^{5 / 2}}-\frac{5 u u^{\prime} u^{\prime \prime} \varphi^{\prime \prime} \eta^{\prime}}{\left(1+\left(u^{\prime}\right)^{2}\right)^{7 / 2}}\right) d x \\
& -\int_{-1}^{1}\left(\frac{5 u^{\prime}\left(u^{\prime \prime}\right)^{2} \varphi^{\prime} \eta}{2\left(1+\left(u^{\prime}\right)^{2}\right)^{7 / 2}}+\frac{5 u\left(u^{\prime \prime}\right)^{2} \varphi^{\prime} \eta^{\prime}}{2\left(1+\left(u^{\prime}\right)^{2}\right)^{7 / 2}}\right) d x \\
& -\int_{-1}^{1}\left(\frac{5 u u^{\prime} u^{\prime \prime} \varphi^{\prime} \eta^{\prime \prime}}{\left(1+\left(u^{\prime}\right)^{2}\right)^{7 / 2}}-\frac{35 u\left(u^{\prime}\right)^{2}\left(u^{\prime \prime}\right)^{2} \varphi^{\prime} \eta^{\prime}}{2\left(1+\left(u^{\prime}\right)^{2}\right)^{9 / 2}}\right) d x \\
& -\frac{1}{2} \int_{-1}^{1}\left(\frac{\varphi^{\prime} \eta^{\prime}}{u\left(1+\left(u^{\prime}\right)^{2}\right)^{3 / 2}}-\frac{u^{\prime} \varphi^{\prime} \eta}{u^{2}\left(1+\left(u^{\prime}\right)^{2}\right)^{3 / 2}}-\frac{3\left(u^{\prime}\right)^{2} \varphi^{\prime} \eta^{\prime}}{u\left(1+\left(u^{\prime}\right)^{2}\right)^{5 / 2}}\right) d x \\
& +\int_{-1}^{1}\left(\frac{u^{\prime \prime} \varphi \eta^{\prime \prime}}{\left(1+\left(u^{\prime}\right)^{2}\right)^{5 / 2}}-\frac{5 u^{\prime}\left(u^{\prime \prime}\right)^{2} \varphi \eta^{\prime}}{2\left(1+\left(u^{\prime}\right)^{2}\right)^{7 / 2}}\right) d x \\
& -\frac{1}{2} \int_{-1}^{1}\left(\frac{-2 \varphi \eta}{u^{3}\left(1+\left(u^{\prime}\right)^{2}\right)^{1 / 2}}-\frac{u^{\prime} \varphi \eta^{\prime}}{u^{2}\left(1+\left(u^{\prime}\right)^{2}\right)^{3 / 2}}\right) d x+\left[\frac{3 u^{\prime} \varphi^{\prime} \eta^{\prime}}{\left(1+\left(u^{\prime}\right)^{2}\right)^{5 / 2}}\right]_{-1}^{1} .
\end{aligned}
$$




\section{B Proof of Lemma 2}

Let us begin by verifying that $\varphi_{1}, \ldots, \varphi_{4}$ as defined in Lemma 2 are indeed solutions of the differential equation in (8). As for $\varphi_{1}$, we compute:

$$
\begin{aligned}
\varphi_{1}^{\prime}(x)= & 2\left(2-x^{2}\right)^{-3 / 2} \\
\varphi_{1}^{\prime \prime}(x)= & 6 x\left(2-x^{2}\right)^{-5 / 2} \\
c(x) \varphi_{1}^{\prime \prime}(x)= & 12 x-6 x\left(2-x^{2}\right)^{1 / 2} \\
\left(c \varphi_{1}^{\prime \prime}\right)^{\prime}(x)= & 12-12\left(2-x^{2}\right)^{1 / 2}+12\left(2-x^{2}\right)^{-1 / 2} \\
\left(c \varphi_{1}^{\prime \prime}\right)^{\prime \prime}(x)= & 12 x\left(2-x^{2}\right)^{-1 / 2}+12 x\left(2-x^{2}\right)^{-3 / 2} ; \\
b(x) \varphi_{1}^{\prime}(x)= & \frac{4}{2-\sqrt{2-x^{2}}}\left(6\left(2-x^{2}\right)^{-1 / 2}+3-10\left(2-x^{2}\right)^{1 / 2}+3\left(2-x^{2}\right)\right) \\
\left(b \varphi_{1}^{\prime}\right)^{\prime}(x)= & -\frac{4 x}{\left(2-\sqrt{2-x^{2}}\right)^{2} \sqrt{2-x^{2}}\left(6\left(2-x^{2}\right)^{-1 / 2}+3-10\left(2-x^{2}\right)^{1 / 2}+3\left(2-x^{2}\right)\right)} \\
& \left.+\frac{4}{2-\sqrt{2-x^{2}}}\left(6 x\left(2-x^{2}\right)^{-3 / 2}+10 x\left(2-x^{2}\right)^{-1 / 2}-6 x\right)\right) \\
= & \frac{4 x}{\left(2-\sqrt{2-x^{2}}\right)^{2}} \\
& \cdot\left(12\left(2-x^{2}\right)^{-3 / 2}-12\left(2-x^{2}\right)^{-1}+17\left(2-x^{2}\right)^{-1 / 2}-12+3\left(2-x^{2}\right)^{1 / 2}\right) \\
= & 12 x\left(2-x^{2}\right)^{-3 / 2}+12 x\left(2-x^{2}\right)^{-1 / 2}+\frac{8 x}{\sqrt{2-x^{2}}\left(2-\sqrt{2-x^{2}}\right)^{2}} \\
= & \left(c \varphi_{1}^{\prime \prime}\right)^{\prime \prime}(x)+a(x) \varphi_{1}(x) .
\end{aligned}
$$

Concerning $\varphi_{2}$, we have:

$$
\begin{aligned}
\varphi_{2}(x) & =1-\frac{1}{\sqrt{2-x^{2}}} \\
\varphi_{2}^{\prime}(x) & =-x\left(2-x^{2}\right)^{-3 / 2} \\
\varphi_{2}^{\prime \prime}(x) & =-\left(2-x^{2}\right)^{-3 / 2}-3 x^{2}\left(2-x^{2}\right)^{-5 / 2} \\
c(x) \varphi_{2}^{\prime \prime}(x) & =-4-4 x^{2}-2\left(2-x^{2}\right)^{3 / 2}+6\left(2-x^{2}\right)^{1 / 2} \\
\left(c \varphi_{2}^{\prime \prime}\right)^{\prime}(x) & =-8 x+6 x\left(2-x^{2}\right)^{1 / 2}-6 x\left(2-x^{2}\right)^{-1 / 2} \\
\left(c \varphi_{2}^{\prime \prime}\right)^{\prime \prime}(x) & =-8+12\left(2-x^{2}\right)^{1 / 2}-12\left(2-x^{2}\right)^{-1 / 2}-12\left(2-x^{2}\right)^{-3 / 2} ; \\
b(x) \varphi_{2}^{\prime}(x) & =-\frac{12 x}{\sqrt{2-x^{2}}\left(2-\sqrt{2-x^{2}}\right)}+\frac{10 x}{2-\sqrt{2-x^{2}}}-8 x+6 x\left(2-x^{2}\right)^{1 / 2} \\
\left(b \varphi_{2}^{\prime}\right)^{\prime}(x)=-\frac{12 x^{2}}{\sqrt{2-x^{2}}\left(2-\sqrt{2-x^{2}}\right)}-\frac{\left.12-x^{2}\right)^{3 / 2}\left(2-\sqrt{2-x^{2}}\right)}{\left(2-x^{2}\right.}+\frac{12 x^{2}}{\left(2-x^{2}\right)\left(2-\sqrt{2-x^{2}}\right)^{2}} & +\frac{10}{2-\sqrt{2-x^{2}}}-\frac{1}{\sqrt{2-x^{2}}\left(2-\sqrt{2-x^{2}}\right)^{2}}-8+6\left(2-x^{2}\right)^{1 / 2}-6 x^{2}\left(2-x^{2}\right)^{-1 / 2} \\
= & \frac{-48+48 \sqrt{2-x^{2}}-20\left(2-x^{2}\right)+8\left(2-x^{2}\right)^{3 / 2}}{\left(2-x^{2}\right)^{3 / 2}\left(2-\sqrt{2-x^{2}}\right)^{2}}-8+12\left(2-x^{2}\right)^{1 / 2}-12\left(2-x^{2}\right)^{-1 / 2} \\
= & \frac{-12\left(2-\sqrt{2-x^{2}}\right)^{2}-8\left(2-x^{2}\right)+8\left(2-x^{2}\right)^{3 / 2}}{\left(2-x^{2}\right)^{3 / 2}\left(2-\sqrt{2-x^{2}}\right)^{2}}+\left(c \varphi_{2}^{\prime \prime}\right)^{\prime \prime}(x)+12\left(2-x^{2}\right)^{-3 / 2} \\
= & \frac{8}{\left(2-\sqrt{2-x^{2}}\right)^{2}}\left(1-\frac{1}{\sqrt{2-x^{2}}}\right)+\left(c \varphi_{2}^{\prime \prime}\right)^{\prime \prime}(x)=a(x) \varphi_{2}(x)+\left(c \varphi_{2}^{\prime \prime}\right)^{\prime \prime}(x) .
\end{aligned}
$$


Concerning $\varphi_{3}$, the calculations are slightly more complicated. For brevity, we denote

$$
Z:=Z(x):=\sqrt{2} \arctan \frac{x}{\sqrt{2}\left(\sqrt{2-x^{2}}-1\right)} .
$$

With the help of

$$
Z^{\prime}(x)=\frac{2}{\sqrt{2-x^{2}}\left(2-\sqrt{2-x^{2}}\right)},
$$

we see that

$$
\begin{aligned}
\varphi_{3}^{\prime}(x)= & -\frac{2}{2-x^{2}} \sin (Z)+\frac{2 x}{\left(2-x^{2}\right)^{3 / 2}} \cos (Z) \\
\varphi_{3}^{\prime \prime}(x)= & \sin (Z)\left(-\frac{4 x}{\left(2-x^{2}\right)^{2}}-\frac{4 x}{\left(2-x^{2}\right)^{2}\left(2-\sqrt{2-x^{2}}\right)}\right) \\
& +\cos (Z)\left(-\frac{2}{\left(2-x^{2}\right)\left(2-\sqrt{2-x^{2}}\right)}-\frac{6}{\left(2-x^{2}\right)^{3 / 2}}+\frac{12}{\left(2-x^{2}\right)^{5 / 2}}\right), \\
c(x) \varphi_{3}^{\prime \prime}(x)= & \sin (Z)\left(-12 x\left(2-x^{2}\right)^{1 / 2}+4 x\left(2-x^{2}\right)\right) \\
& +\cos (Z)\left(-4\left(2-x^{2}\right)\left(2-\sqrt{2-x^{2}}\right)+12\left(2-\sqrt{2-x^{2}}\right)-4\left(2-x^{2}\right)\right), \\
\left(c \varphi_{3}^{\prime \prime}\right)^{\prime}(x)= & \sin (Z)\left(\frac{16}{2-\sqrt{2-x^{2}}}-12 x^{2}-16 \sqrt{2-x^{2}}\right) \\
& +\cos (Z)\left(-\frac{8 x}{2-\sqrt{2-x^{2}}}+\frac{12 x}{\sqrt{2-x^{2}}}+16 x-12 x \sqrt{2-x^{2}}\right) \\
b(x) \varphi_{3}^{\prime}(x)= & \sin (Z)\left(\frac{16}{2-\sqrt{2-x^{2}}+4}-12 x^{2}-16 \sqrt{2-x^{2}}\right) \\
& +\cos (Z)\left(-\frac{8 x}{2-\sqrt{2-x^{2}}}+\frac{12 x}{\sqrt{2-x^{2}}}+16 x-12 x \sqrt{2-x^{2}}\right),
\end{aligned}
$$

so that finally

$$
\begin{aligned}
\left(c \varphi_{3}^{\prime \prime}\right)^{\prime}(x)-b(x) \varphi_{3}^{\prime}(x) & =-4 \sin (Z) \\
\left(\left(c \varphi_{3}^{\prime \prime}\right)^{\prime}-b \varphi_{3}^{\prime}\right)^{\prime}(x) & =-\frac{8}{\sqrt{2-x^{2}}\left(2-\sqrt{2-x^{2}}\right)} \cos (Z)=-a(x) \varphi_{3}(x) .
\end{aligned}
$$

The calculations for $\varphi_{4}$ are similar:

$$
\begin{aligned}
\varphi_{4}^{\prime}(x)= & \frac{2}{2-x^{2}} \cos (Z)+\frac{2 x}{\left(2-x^{2}\right)^{3 / 2}} \sin (Z), \\
\varphi_{4}^{\prime \prime}(x)= & \cos (Z)\left(\frac{4 x}{\left(2-x^{2}\right)^{2}}+\frac{4 x}{\left(2-x^{2}\right)^{2}\left(2-\sqrt{2-x^{2}}\right)}\right) \\
& +\sin (Z)\left(-\frac{2}{\left(2-x^{2}\right)\left(2-\sqrt{2-x^{2}}\right)}-\frac{6}{\left(2-x^{2}\right)^{3 / 2}}+\frac{12}{\left(2-x^{2}\right)^{5 / 2}}\right), \\
c(x) \varphi_{4}^{\prime \prime}(x)= & \cos (Z)\left(12 x\left(2-x^{2}\right)^{1 / 2}-4 x\left(2-x^{2}\right)\right) \\
& +\sin (Z)\left(-4\left(2-x^{2}\right)\left(2-\sqrt{2-x^{2}}\right)+12\left(2-\sqrt{2-x^{2}}\right)-4\left(2-x^{2}\right)\right), \\
\left(c \varphi_{4}^{\prime \prime}\right)^{\prime}(x)= & \cos (Z)\left(-\frac{16}{2-\sqrt{2-x^{2}}}+12 x^{2}+16 \sqrt{2-x^{2}}\right) \\
& +\sin (Z)\left(-\frac{8 x}{2-\sqrt{2-x^{2}}}+\frac{12 x}{\sqrt{2-x^{2}}}+16 x-12 x \sqrt{2-x^{2}}\right)
\end{aligned}
$$




$$
\begin{aligned}
b(x) \varphi_{4}^{\prime}(x)= & \cos (Z)\left(-\frac{16}{2-\sqrt{2-x^{2}}}-4+12 x^{2}+16 \sqrt{2-x^{2}}\right) \\
& +\sin (Z)\left(-\frac{8 x}{2-\sqrt{2-x^{2}}}+\frac{12 x}{\sqrt{2-x^{2}}}+16 x-12 x \sqrt{2-x^{2}}\right)
\end{aligned}
$$

so that finally

$$
\begin{aligned}
\left(c \varphi_{4}^{\prime \prime}\right)^{\prime}(x)-b(x) \varphi_{4}^{\prime}(x) & =4 \cos (Z) \\
\left(\left(c \varphi_{4}^{\prime \prime}\right)^{\prime}-b \varphi_{4}^{\prime}\right)^{\prime}(x) & =-\frac{8}{\sqrt{2-x^{2}}\left(2-\sqrt{2-x^{2}}\right)} \sin (Z)=-a(x) \varphi_{4}(x)
\end{aligned}
$$

It remains to prove that $\varphi_{1}, \ldots, \varphi_{4}$ are linearly independent. We shall present the argument in such a way that it can be also used to prove Lemma 3 . Assume that $\sum_{i=1}^{4} \beta_{i} \varphi_{i} \equiv 0$ in $[-1,1]$. Then we have in particular

$$
\begin{aligned}
\sum_{i=1}^{4} \beta_{i} \varphi_{i}(1)=\sum_{i=1}^{4} \beta_{i} \varphi_{i}(-1) & =0 \\
\sum_{i=1}^{4} \beta_{i} \varphi_{i}^{\prime \prime}(1)-2 \sum_{i=1}^{4} \beta_{i} \varphi_{i}^{\prime}(1)=0, \sum_{i=1}^{4} \beta_{i} \varphi_{i}^{\prime \prime}(-1)+2 \sum_{i=1}^{4} \beta_{i} \varphi_{i}^{\prime}(-1) & =0 .
\end{aligned}
$$

We need the following values of $\varphi_{i}$ and its derivatives:

$$
\begin{aligned}
& \varphi_{1}(1)=-\varphi_{1}(-1)=1, \\
& \varphi_{1}^{\prime \prime}(1)=-\varphi_{1}^{\prime \prime}(-1)=6 \text {, } \\
& \varphi_{2}(1)=\varphi_{2}(-1)=0 \text {, } \\
& \varphi_{2}^{\prime \prime}(1)=\varphi_{2}^{\prime \prime}(-1)=-4 \text {, } \\
& \varphi_{3}(1)=\varphi_{3}(-1)=\cos \left(\frac{\sqrt{2}}{2} \pi\right) \text {, } \\
& \varphi_{1}^{\prime}(1)=\varphi_{1}^{\prime}(-1)=2, \\
& \varphi_{3}^{\prime \prime}(1)=\varphi_{3}^{\prime \prime}(-1)=4 \cos \left(\frac{\sqrt{2}}{2} \pi\right)-8 \sin \left(\frac{\sqrt{2}}{2} \pi\right) \text {, } \\
& \varphi_{4}(1)=-\varphi_{4}(-1)=\sin \left(\frac{\sqrt{2}}{2} \pi\right), \quad \varphi_{4}^{\prime}(1)=\varphi_{4}^{\prime}(-1)=2 \cos \left(\frac{\sqrt{2}}{2} \pi\right)+2 \sin \left(\frac{\sqrt{2}}{2} \pi\right) \text {, } \\
& \varphi_{4}^{\prime \prime}(1)=-\varphi_{4}^{\prime \prime}(-1)=8 \cos \left(\frac{\sqrt{2}}{2} \pi\right)+4 \sin \left(\frac{\sqrt{2}}{2} \pi\right), \\
& \varphi_{2}^{\prime}(1)=-\varphi_{2}^{\prime}(-1)=-1, \\
& \varphi_{3}^{\prime}(1)=-\varphi_{3}^{\prime}(-1)=2 \cos \left(\frac{\sqrt{2}}{2} \pi\right)-2 \sin \left(\frac{\sqrt{2}}{2} \pi\right),
\end{aligned}
$$

With the help of these values, we conclude from (32) that

$$
\beta_{3}=0, \quad \beta_{1}=-\beta_{4} \sin \left(\frac{\sqrt{2}}{2} \pi\right) .
$$

Combining this result with (33) we infer

$$
\left\{\begin{array}{l}
0=-\beta_{2}+\beta_{4}\left(2 \cos \left(\frac{\sqrt{2}}{2} \pi\right)-\sin \left(\frac{\sqrt{2}}{2} \pi\right)\right) \\
0=-\beta_{2}-\beta_{4}\left(2 \cos \left(\frac{\sqrt{2}}{2} \pi\right)-\sin \left(\frac{\sqrt{2}}{2} \pi\right)\right),
\end{array}\right.
$$

which by $\frac{\sqrt{2}}{2} \pi \in\left(\frac{\pi}{2}, \pi\right),\left(2 \cos \left(\frac{\sqrt{2}}{2} \pi\right)-\sin \left(\frac{\sqrt{2}}{2} \pi\right)\right)<0$ gives

$$
0=\beta_{2}=\beta_{4}
$$

Finally, from (34) we obtain that also $\beta_{3}=\beta_{1}=0$.

Acknowledgement. We are grateful to our colleague A. Dall'Acqua for fruitful discussions, for carefully reading the manuscript, and for pointing out an error in preliminary calculations. 


\section{References}

[BK] M. Bauer, E. Kuwert, Existence of minimizing Willmore surface of prescribed genus, Int. Math. Res. Not. 2003, No.10, 553-576 (2003).

[CR] M.G. Crandall, P.H. Rabinowitz, Bifurcation from simple eigenvalues, J. Functional Analysis 8, 321-340 (1971).

[DDG] A. Dall'Acqua, K. Deckelnick, H.-Ch. Grunau, Classical solutions to the Dirichlet problem for Willmore surfaces of revolution, Adv. Calc. Var. 1, 379-397 (2008).

[DFGS] A. Dall'Acqua, S. Fröhlich, H.-Ch. Grunau, F. Schieweck, Symmetric Willmore surfaces of revolution satisfying arbitrary Dirichlet boundary data, Preprint 24-2008, Faculty of Mathematics, University of Magdeburg, 2008.

[De] K. Deimling, Nonlinear functional analysis, Springer-Verlag, Berlin etc. (1985).

[DHKW] U. Dierkes, S. Hildebrandt, A. Küster, O. Wohlrab, Minimal Surfaces I, Grundlehren der mathematischen Wissenschaften 295, Springer-Verlag: Berlin etc. (1992).

[F] S. Fröhlich, Katenoidähnliche Lösungen geometrischer Variationsprobleme, Preprint 2322, FB Mathematik, TU Darmstadt, 2004.

[Ka] D. Kastsian, Finite element approximation of two-dimensional rotationally symmetric Willmore surfaces, Master's thesis, University of Magdeburg, 2007.

[KS1] E. Kuwert, R. Schätzle, Closed surfaces with bounds on their Willmore energy, Preprint, 2008.

[KS2] E. Kuwert, R. Schätzle, Minimizers of the Willmore functional under fixed conformal class, Preprint, 2008.

[LPP $\quad$ K. Leschke, F. Pedit, U. Pinkall, Willmore tori in the 4-sphere with nontrivial normal bundle, Math. Ann. 332, 381-394 (2005).

[Nit] J.C.C. Nitsche, Boundary value problems for variational integrals involving surface curvatures, Quarterly Appl. Math. 51, 363-387 (1993).

[P] S.D. Poisson, Mémoire sur les surfaces élastiques, Cl. Sci. Mathém. Phys. Inst. de France, 2nd printing, 167-225 (1812).

[R] T. Rivière, Analysis aspects of Willmore surfaces, Invent. Math. 174, 1-45 (2008).

[Sch] R. Schätzle, The Willmore boundary value problem, Preprint, 2006.

[Sn] L. Simon, Existence of surfaces minimizing the Willmore functional, Commun. Anal. Geom. 1, 281-326 (1993).

[Th] G. Thomsen, Über konforme Geometrie I: Grundlagen der konformen Flächentheorie, Abh. Math. Sem. Univ. Hamburg 3, 31-56 (1923).

[Wi] T.J. Willmore, Riemannian geometry, Oxford Science Publications, The Clarendon Press, Oxford University Press, New York, 1993. 